\title{
Emerging importance of satellite glia in nervous system function and dysfunction
}

\section{Menachem Hanani ${ }^{1 凶}{ }^{\otimes}$ and David C. Spray $\mathbb{1}^{2}$}

Abstract | Satellite glial cells (SGCs) closely envelop cell bodies of neurons in sensory, sympathetic and parasympathetic ganglia. This unique organization is not found elsewhere in the nervous system. SGCs in sensory ganglia are activated by numerous types of nerve injury and inflammation. The activation includes upregulation of glial fibrillary acidic protein, stronger gap junctionmediated SGC-SGC and neuron-SGC coupling, increased sensitivity to ATP, downregulation of Kir4.1 potassium channels and increased cytokine synthesis and release. There is evidence that these changes in SGCs contribute to chronic pain by augmenting neuronal activity and that these changes are consistent in various rodent pain models and likely also in human pain. Therefore, understanding these changes and the resulting abnormal interactions of SGCs with sensory neurons could provide a mechanistic approach that might be exploited therapeutically in alleviation and prevention of pain. We describe how SGCs are altered in rodent models of four common types of pain: systemic inflammation (sickness behaviour), post-surgical pain, diabetic neuropathic pain and post-herpetic pain.

Satellite glial cells (SCCs). Glial cells that surround neurons in sensory, sympathetic and parasympathetic ganglia (they should not be confused with satellite cells, which are the progenitor cells in striated muscles).
'Laboratory of Experimental Surgery, Hadassah-Hebrew University Medical Center and Faculty of Medicine, Hebrew University of Jerusalem, Jerusalem, Israel.

${ }^{2}$ Dominick Purpura

Department of Neuroscience, Albert Einstein College of

Medicine, New York, NY, USA.

凶e-mail:hananim@

cc.huji.ac.il

https://doi.org/10.1038/

\$41583-020-0333-2
Satellite glial cells (SGCS) are unique cells whose most distinctive morphological feature is that they wrap around neuronal cell bodies, in most cases forming a complete envelope. SGCs are found exclusively in peripheral ganglia - sensory, parasympathetic and sympathetic ganglia (FIG. 1a,b). The latter two are part of the autonomic nervous system. The gap between SGCs and the neuronal surface is about $20 \mathrm{~nm}$, which is similar to that of the synaptic cleft. This organization allows for close mutual neuron-SGC interactions ${ }^{1-3}$. The neuron and its attendant SGCs have been termed 'neuron-glial units' (FIG. 1 C). In most cases, neurons are wrapped individually with several SGCs, but there is evidence that a small proportion (4-9\% depending on the species) of dorsal root ganglia (DRG) neurons share a common glial envelope, forming a 'cluster' with one or two other neurons ${ }^{4}$ (FIG. 1 d). In some cases, the neurons in a cluster are separated only by a thin layer of extracellular space, whereas in other cases they are separated by a thin glial sheet, which allows neuron-SGC-neuron chemical interaction ${ }^{5}$.

Much more is known about astrocytes than SGCs, so it is instructive to compare SGCs with these as well as with other types of glia (BOX 1). Two main hallmarks of astrocyte-neuron relations are that each astrocyte contacts numerous neurons, and that individual astrocytes form non-overlapping domains ${ }^{6}$. In contrast,
SGCs around a given neuron are in close contact with each other, and are almost always separated from SGCs surrounding other neurons.

The close contact between SGCs and neurons is a major key to understanding their functions; for example, this contact enables them to control neuronal homeostasis, but very little is known on this topic. More is known with regard to changes that SGCs in sensory ganglia undergo following nerve damage and how they contribute to pain. Little is known about the functions of SGGs in sympathetic ganglia, but there is emerging information on their role in the control of synaptic transmission in these ganglia. Knowledge of SGCs in parasympathetic ganglia is even scarcer.

SGCs and pain. Glial cells play major roles in the function of the CNS under health and disease states, and maintain bidirectional communication with the neurons ${ }^{7-9}$. Glial cells express receptors for substances released from neurons, most notably ATP, acting on P2 purinergic receptors (P2Rs) ${ }^{10,11}$. Glial cells also release bioactive substances, including ATP, glutamate and cytokines such as tumour necrosis factor (TNF), IL- $1 \beta$ and fractalkine ${ }^{7,12}$, which enable them to communicate among themselves and with adjacent neurons. Most studies on pain and most treatments for it have focused on neurons, but Watkins and her co-workers ${ }^{13,14}$ proposed that glial cells in the spinal 


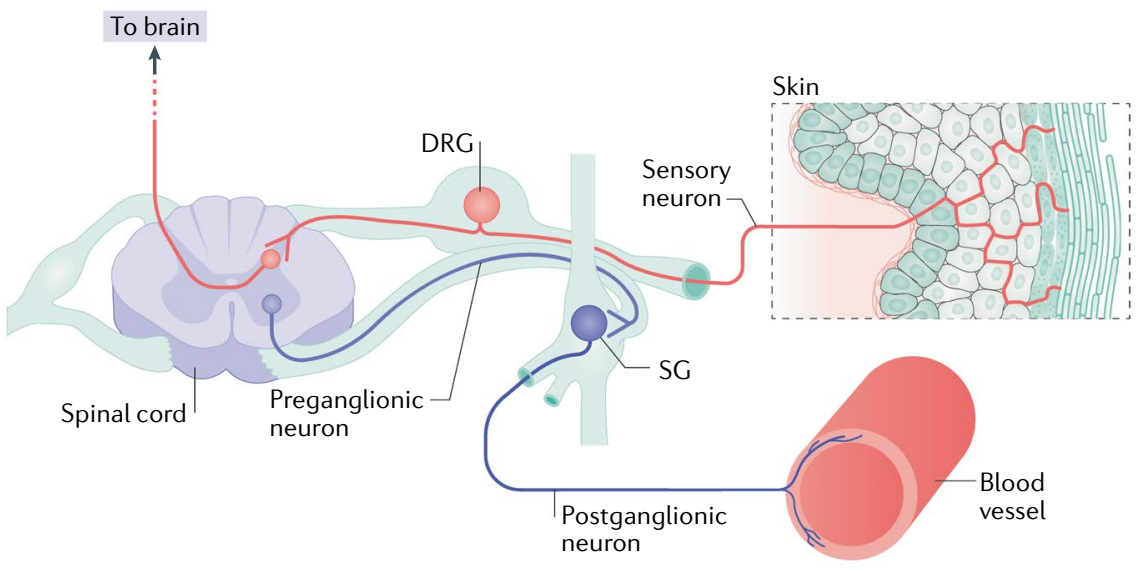

b

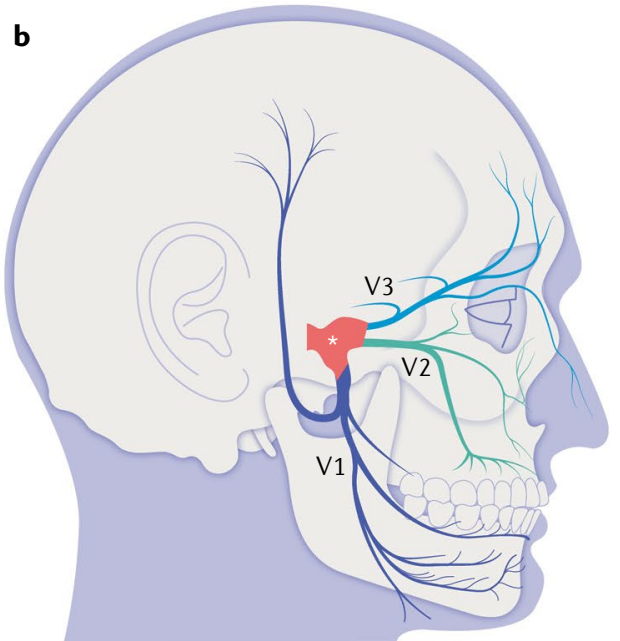

C

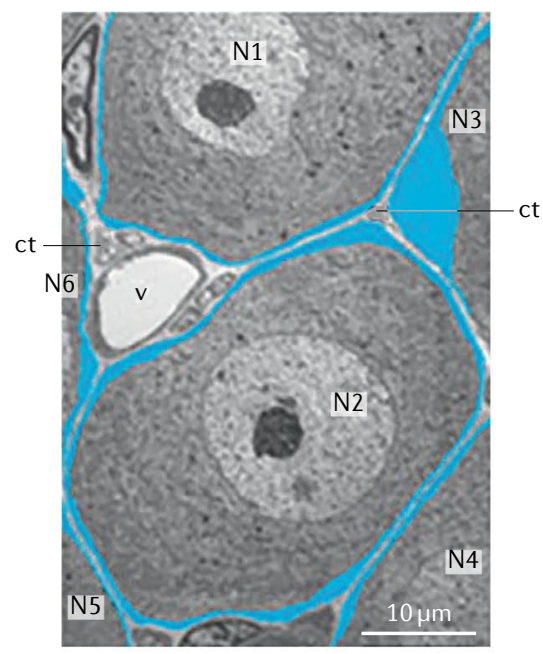

d

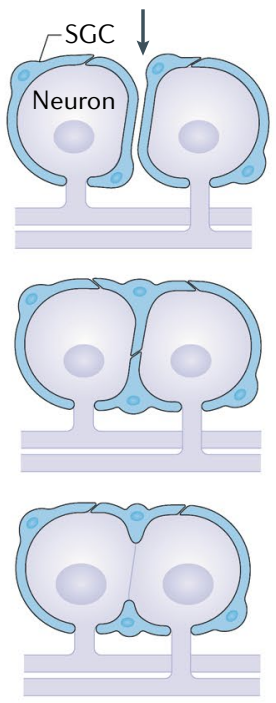

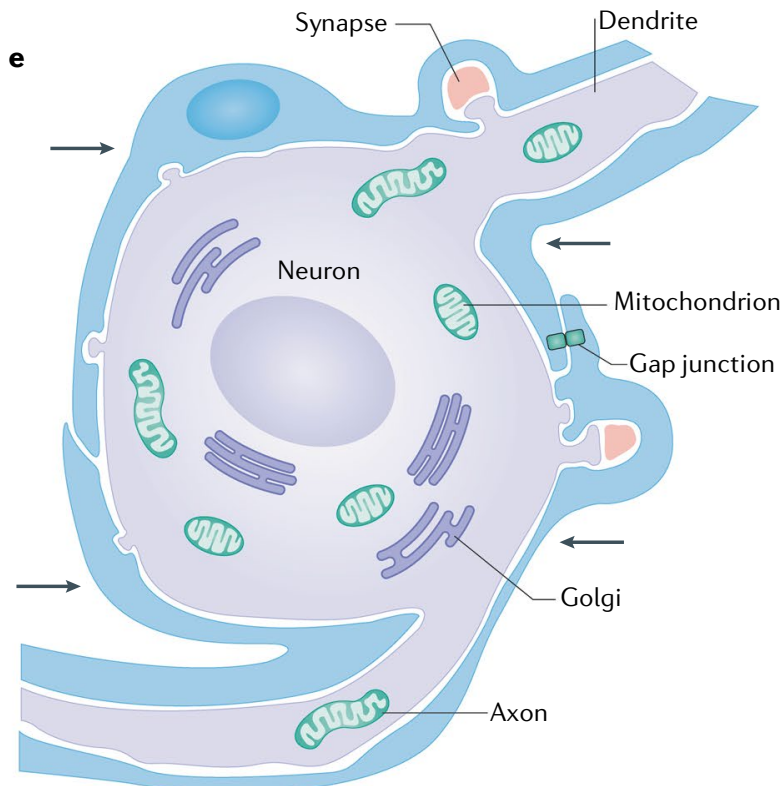

Fig. 1 | Location and morphology of SGCs. a | Position of the dorsal root ganglia (DRG) in the sensory pathways leading from the skin to the brain. A paravertebral sympathetic ganglion (SG) is also indicated. These ganglia innervate most organs, including blood vessels. $\mathbf{b}$ | Location of the trigeminal ganglion (asterisk) which innervates the face and teeth. The three divisions of the trigeminal ganglion are indicated as V1-V3. c | Low-power electron micrograph showing the neuron-satellite glial cell (SGC) units in a DRG. Neurons are labelled N1-N6, SGCs are coloured blue. The widened area in the SGC surrounding N3 contains the cell's nucleus. ct, connective tissue space; v, blood vessels. Scale bar, $10 \mu \mathrm{m}$. d) Schematic of three patterns of grouping of sensory neurons. Top: neurons are separated by a connective tissue space (indicated by arrow), and each has its own SGC sheath. Middle: a cluster of two neurons that share a common SGC sheath and are separated by a SGC process. Bottom: a cluster where the neurons share a common SGC sheath, but without an intervening SGC process. e Schematic of a sympathetic neuron covered with an SGC envelope. The SGCs (arrows) cover the synapses. SGC processes extend beyond the neuronal soma and ensheath an axon and a dendrite. Part c adapted with permission from REF. ${ }^{86}$, Elsevier. Part e adapted with permission from REF. ${ }^{127}$, Elsevier.

\section{Sympathetic ganglia}

Clusters of neuron cell bodies

that innervate smooth muscles, heart and glands; paravertebra ganglia are arranged along the spinal column, and

prevertebral ones are located in the abdomen. cord participate in pain generation and maintenance, and may serve as more suitable targets for pain therapy. This view was supported by studies of pain models in animals, showing that spinal microglia and astrocytes are essential for hyperalgesia ${ }^{7,15,16}$.

Sensory ganglia contain the somata of neurons that innervate most body parts. The main sensory ganglia are the DRG, trigeminal ganglia (TG) and nodose ganglia (FIG. 1). There is evidence that abnormal electrical activity in sensory neurons is associated with pathological pain, such as allodynia ${ }^{17-20}$. For example, it has been shown that phantom limb pain in humans is driven primarily by abnormal intrinsic DRG activity ${ }^{21}$. The ideas described above concerning CNS glia and pain prompted research into the possible role of SGCs in sensory ganglia in pain. It was found that nerve damage or inflammation activates SGCs in sensory ganglia. This included upregulation of the astrocyte marker glial fibrillary acidic 
Dorsal root ganglia

(DRG). Clusters of cells located near the spinal cord containing the cell bodies of peripheral neurons that innervate most body parts, including internal organs.

\section{Sensory ganglia}

Clusters of neuron cell bodies

that have a single axon that bifurcates to two branches; one branch runs to the periphery and can detect various stimuli, and the other projects into the central nervous system.

P2 purinergic receptors (P2Rs). Receptors for the neurotransmitter adenosine (P1) and ATP (P2). There are seven ionotropic receptors (P2X1-P2X7) and eight

$\mathrm{C}$ protein-coupled receptors (P2Y1, P2Y2, P2Y4, P2Y6, P2Y11-P2Y14]. protein (GFAP) ${ }^{22-24}$, increased coupling strength among SGCs $^{19,25-27}$, downregulated Kir4. 1 channels in SGCs ${ }^{28-30}$ and increased sensitivity of SGCs to the pain mediator $\operatorname{ATP}^{27,31}$ (see BOX 2 for details). Activated SGCs release pro-inflammatory cytokines IL- $1 \beta$, IL- 6 , TNF and fractalkine ${ }^{2,32-36}$, which can act on the neurons and increase their excitability and firing.

In this Review, we describe the normal properties of SGCs and how they change in animal pain models. We discuss the possible roles of SGCs in several models of pain in rodents - systemic inflammation, postsurgical pain (PSP), diabetic neuropathic pain (DNP) and post-herpetic pain. These studies indicate that these different pain syndromes share a rather similar chain of events that connects changes in SGCs to pain. Essentially, nerve injury leads to nitric oxide release, which induces SGC activation. SGCs in turn release compounds that lead to neuronal hyperexcitability and pain. Intercellular calcium waves play an important role in this scheme. We conclude with a brief account of SGCs in sympathetic ganglia and their possible roles under pathological conditions.

\section{Characteristics of SGCs}

Ion channels. SGCs do not have voltage-dependent $\mathrm{Na}^{+}$ or $\mathrm{Ca}^{2+}$ channels, and therefore cannot conduct action potentials ${ }^{37}$. The main $\mathrm{K}^{+}$channel in SGCs is Kir4.1 $\left(R^{2}{ }^{28}\right.$ ). Injury suppresses Kir4.1 function in SGCs, which may contribute to pain ${ }^{29,30}$. Reduced Kir4.1 permeability likely depolarizes SGCs, inducing them to release excitatory mediators such as ATP that can activate the neurons; indeed, silencing Kir4.1 in the TG leads to pain-like behaviour ${ }^{28}$. Similarly, gain or loss of Kir4.1 in astrocytes that contact the somata of CNS neurons can regulate neuronal activity ${ }^{38}$.

Gap junctions in SGCs. Glial cells in both the CNS and the periphery express gap junction channels, which provide a pathway for diffusion of ions and small molecules between cells. The most abundant connexins $(\mathrm{Cx})$ in mouse DRG and TG are $\mathrm{Cx} 43$ and $\mathrm{Cx} 32$, followed by $\mathrm{Cx} 30.2, \mathrm{Cx} 37, \mathrm{Cx} 26, \mathrm{Cx} 30, \mathrm{Cx} 45$ and $\mathrm{Cx} 36\left(\mathrm{REF}^{39}\right)$. Under normal conditions, SGCs contain Cx43 (REFS ${ }^{40,41}$ ). Cx43 is upregulated in the TG and DRG following nerve injury or inflammation ${ }^{26,41-45}$. Cx26 expression

\section{Box 1 | What sorts of glia are SGCs?}

Satellite glial cells (SGCs) exhibit similarities and differences compared with CNS astrocytes and oligodendrocytes:

- Morphology: whereas astrocytes and oligodendrocytes are highly branched and polarized, SGCs are flattened (see the figure, parts a,b). There is no evidence for contacts between SGCs and blood vessels, analogous to the endfeet that astrocytes make with blood vessels, and unlike oligodendrocytes, SGCs do not myelinate (with the exception of those in spiral ganglia).

- Microenvironment control: astrocyte transporters and ion channels control glutamate and $\mathrm{K}^{+}$levels at synapses. SGCs express $\mathrm{K}^{+}$channels and glutamate transporters, and probably perform similar control and protective functions ${ }^{1}$.

- Electrical coupling: both astrocytes and SGCs are interconnected by gap junctions. This coupling is stronger and more extensive in astrocytes than in SGCs, but in both cell types, coupling increases following damage.

- Molecular markers: SGCs share marker molecules with all types of CNS glia and represent an intermediate type between astrocytes and oligodendrocytes ${ }^{143}$. Like astrocytes, SGCs express glutamine synthetase, S100, vimentin, glutamate aspartate transporter (GLAST), connexin 43 (Cx43) and Kir4.1 potassium channels ${ }^{1,3,26,28}$.

- Lineage and plasticity: SGCs are derived from the neural crest. They contain cadherin 19, which is a Schwann cell marker ${ }^{144}$, and it was proposed that SGCs represent a subpopulation of cells in the Schwann cell lineage whose further differentiation is arrested due to contact with sensory neuron somata ${ }^{145}$. Adult $\mathrm{SGC}$ s express the transcription factor Sox2, which is typical for stem cells ${ }^{146}$. Consistent with this, SGCs can function as progenitor cells, giving rise to neurons ${ }^{147,148}$, Schwann cells, astrocytes and oligodendrocytes ${ }^{149}$. Cultured SGCs migrate from the neurons, change their phenotype and assume Schwann cell-like morphology ${ }^{150}$. Like oligodendrocytes, SGCs express the transcription factor Sox10 and several early myelination markers ${ }^{151}$. Sox 10 can convert SGCs towards the oligodendrocyte phenotype ${ }^{151}$. SGC responses to injury, such as growing new processes ${ }^{25,27}$, may be related to this plasticity

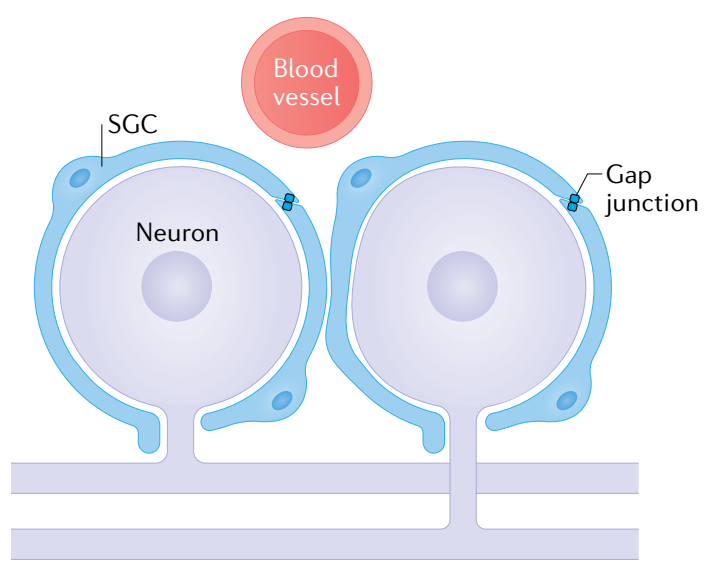

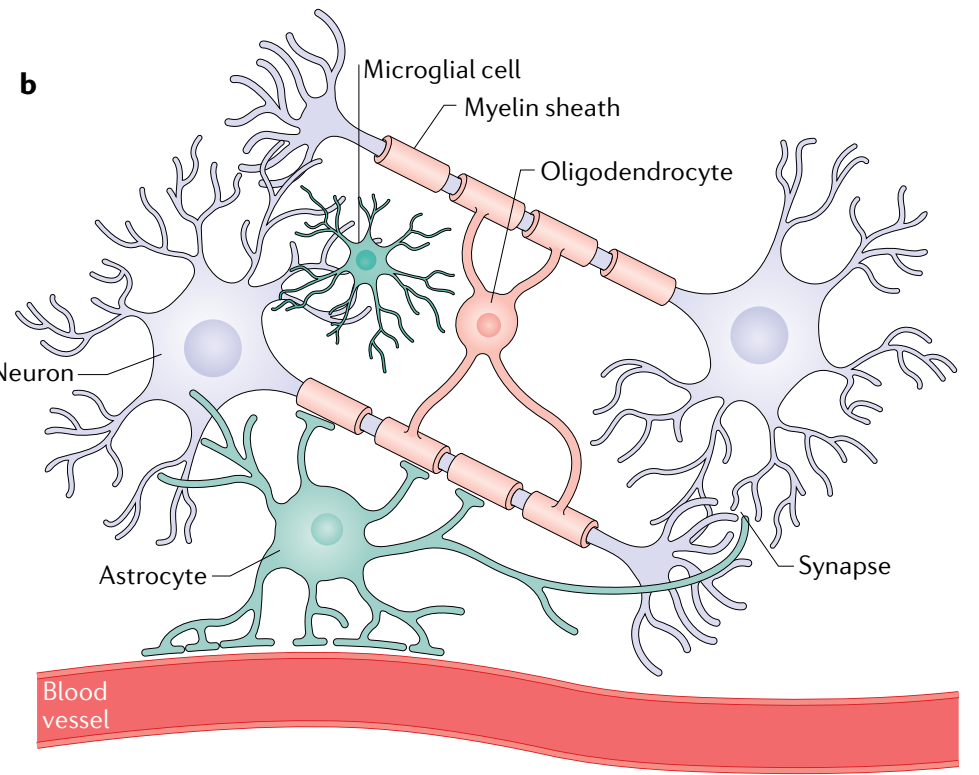




\section{Box 2 | Glial activation (gliosis)}

The concept of glial activation derives from the field of immune cell activation. In the presence of danger signals, immune cells transform into an activated state, which is manifested by increased cytokine production, action as antigen-presenting cells and phagocytosis of invading cells. Glial cells, and in particular microglia, can function as immune cells, and it was proposed that activation of glial cells in the spinal cord is a key process in chronic pain ${ }^{7,13}$. A hallmark of astrocyte activation is an increased glial fibrillary acidic protein (GFAP) expression, and it was found that peripheral injury also induced this effect in satellite glial cells (SGCs) in sensory ganglia ${ }^{24}$. Moreover, SGCs in human trigeminal ganglia act as antigen-presenting cells and are able to phagocytose cells ${ }^{152}$, and SGC precursors phagocytose dead neurons during development in mouse dorsal root ganglia (DRG) ${ }^{153}$. SGCs express macrophage markers, such as MHC type II, CD40 and CD54, but not others (CD14 and CD68), and thus appear to have a unique leukocyte phenotype ${ }^{152}$. It was proposed that SGC activation includes elevated GFAP, increased coupling by gap junctions and elevated sensitivity to ATP ${ }^{80}$. Increased production of pro-inflammatory cytokines in activated SGCs was also noted ${ }^{2,32-36}$. Disruption of neuronal p75NTR expression induced higher levels of GFAP, p75NTR and connexin 43 ( $C \times 43)$, and lower levels of Kir4.1 protein in $\mathrm{SGCs}^{154}$. Thus, the definition of SGC activation extends beyond GFAP upregulation.

The increased coupling between SGCs following injury is of special interest, as this can contribute to the spread and enhancement of neuronal excitation. One way of assessing coupling is the dye coupling method. Under resting conditions (see the figure, part a), SGCs are dye-coupled to other SGCs around the same neuron. The SGC marked with an asterisk was injected with the fluorescent dye Lucifer yellow, which crosses gap junctions, and the dye spread to three other SGCs. In the DRG of a mouse treated with the chemotherapeutic drug oxaliplatin, which causes neuropathic pain, dye spread occurs also to SGCs around adjacent neurons (see the figure, part b). These results correlate with electron-microscopic studies that showed increased numbers of gap junctions between SGCs following injury or inflammation. Scale bar, $20 \mu \mathrm{m}$. After nerve injury (see the figure, part c), SGCs that are part of the glial sheaths of two distinct neurons ( $\mathrm{N} 1$ and $\mathrm{N} 2$ ) form a bridge where they are connected by gap junctions. The boxed area in part $\mathbf{c}$ (in the outlined area) is also shown at higher enlargement (see the figure, part $\mathbf{d}$ ), where a gap junction is
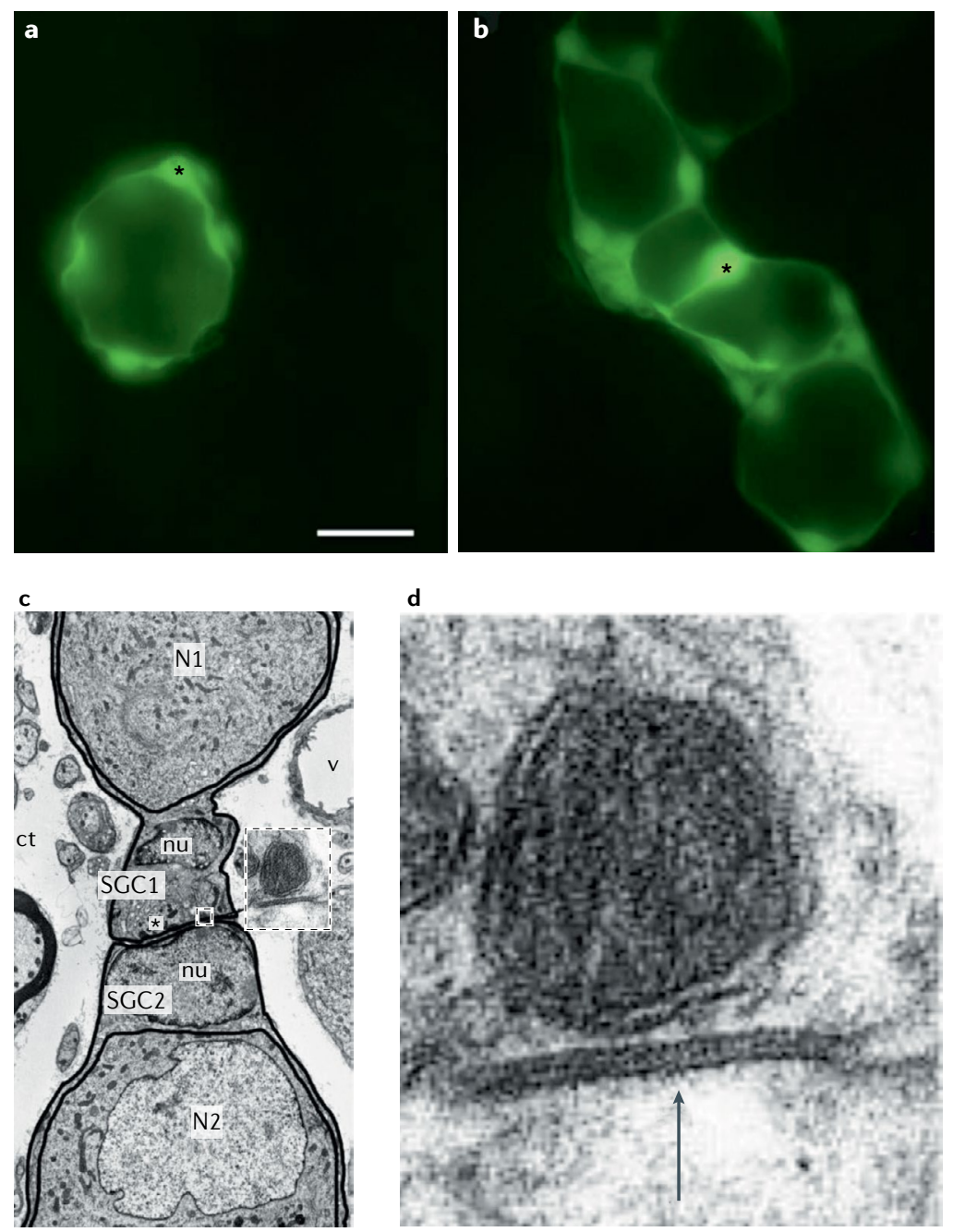

indicated with an arrow. Asterisk indicates an unmyelinated axon. ct, connective tissue space; $v$, blood vessel; nu, nuclei of SGCs. Mouse DRG is ipsilateral to sciatic nerve axotomy. Figure, parts $\mathbf{a}, \mathbf{b}$ adapted with permission from REF. ${ }^{23}$, Wiley. Part c adapted from REF. ${ }^{47}$, Springer Nature Limited.
Trigeminal ganglia (TC). Clusters of cells located at the base of the skull (but outside the brain) that contain the cell bodies of neurons that innervate the face, teeth and scalp.

\section{Nodose ganglia}

Clusters of neuron cell bodies that innervate many visceral organs, such as the intestine and heart.

\section{Allodynia}

Pain resulting from a non-

noxious stimulus to normal skin. in SGCs and neurons was increased in the TG in the setting of neuropathic pain ${ }^{46}$. These findings correlate with functional evidence for increased coupling by gap junctions among SGCs in a wide range of pain models, which include the sciatic nerve section ${ }^{25,47}$, the infraorbital nerve section ${ }^{37}$, colonic obstruction and inflammation ${ }^{19,48,49}$, injection of complete Freund's adjuvant in mouse hindpaw ${ }^{50}$, the sciatic nerve ${ }^{51}$, the temporomandibular joint ${ }^{52}$ and chemotherapy-induced neuropathy ${ }^{23}$. Dye coupling among the SGCs not only increased within the sheaths surrounding individual neurons but extended to sheaths surrounding adjacent neurons via newly formed processes ${ }^{19,23,25,27,47,51,52}$. Moreover, injury generally results in dye spread between SGCs and neurons and from neuron to neuron, and electrophysiological recordings from the dissociated DRG and TG demonstrate electrical coupling through SGC-SGC, neuron-SGC and neuron-neuron gap junctions $^{44,53}$.
As SGC activation is accompanied with increased coupling, it was proposed that blocking gap junctions could have an analgesic action, and this was confirmed in numerous chronic pain models ${ }^{19,23,26,42-45,50,54,55}$. Reduced Cx43 in rat TG using double-stranded RNA was analgesic in a model of neuropathic pain induced by infraorbital nerve injury ${ }^{26}$, although a potential concern was that in naive rats this treatment itself induced pain-like behaviours. A recent study has shown that increased SGC coupling contributes also to acute pain induced by capsaicin application ${ }^{56}$. Blocking gap junctions with carbenoxolone (CBX) or blocking P2X7Rs (which are expressed exclusively by SGCs) reduced pain reactions in this model, supporting a role for SGCs in acute pain.

In summary, increased coupling between SGCs and also SGC-neuron and neuron-neuron coupling is a common feature in pain models, and appears to contribute to chronic pain. We propose that inhibiting gap 
Kir4. 1 channels

Inward rectifier channels that

tend to favour the influx of

potassium ions into cells over

their efflux

Gap junctions

Intercellular channels that

provide a pathway for diffusion

of ions and small molecules

between cells; they are made

of connexin $(\mathrm{Cx})$ proteins.

Dye coupling

A method for studying gap

junction-mediated coupling

between cells, based on

injecting a cell with a dye that

passes these junctions and

examining whether the dye

passed to nearby cells. junctions might be an effective novel target for analgesic drugs.

Pannexins in SGCs. Pannexins (Panx) are homologues of gap junction proteins of multicellular invertebrates, the innexins. Although Panx1 does not form gap junctions, it does form membrane channels that allow release of $\mathrm{ATP}^{57,58}$. Panx1 expression is upregulated in the DRG following sciatic nerve ligation ${ }^{59}$, in TG SGCs in a chronic orofacial pain model ${ }^{60}$ and in nodose ganglia SGCs in a systemic inflammation model ${ }^{61}$. In the orofacial pain study ${ }^{60}$, Panx1-deleted mice did not develop tactile hypersensitivity, suggesting a role for Panx1-mediated ATP release in nociception. This analgesic effect was obtained in mice with glia-targeted but not with neuronspecific Panx1 deletion ${ }^{60}$, indicating the dominant role of SGCs in ATP release.

Receptors. Knowledge regarding the pharmacology of SGCs is still limited, and much of the information on this topic relates to purinergic transmission. ATP is a messenger in neuron-glia interactions ${ }^{11}$ and in the pain pathways ${ }^{15,62}$. Cells release ATP by vesicular or channelmediated mechanisms, such as P2X7R or Panx1 channels. Ecto-ATPases break down ATP to ADP and other purines. In the DRG, ecto-ATPases are found in Schwann cells and SGCs, but not in neurons ${ }^{63}$. This enables these glial cells to regulate the ATP level in the ganglia.

Using calcium imaging, SGCs in the TG were found to express functional P2YRs (REF. ${ }^{64}$ ). Further work showed that P2Rs in the TG consist of P2Y1, P2Y2, P2Y4, P2Y6, $\mathrm{P} 2 \mathrm{Y} 12$ and P2Y13Rs (REFS ${ }^{62,65}$ ). In vitro incubation with the pro-inflammatory peptide bradykinin increased the SGC response to P2YR stimulation ${ }^{65}$, indicating P2YR sensitization. Release of ATP by sensory neurons can activate P2Rs in SGCs, providing one mechanism for neuron-SGC communication ${ }^{2,5,36,66-69}$ (FIG. 2a,b). In two orofacial pain models, the sensitivity of SGCs to ATP in the TG increased 100 -fold ${ }^{31}$, apparently due to a switch of the P2R population from the metabotropic P2YR to the ionotropic P2XR. The exact P2XR subtype that was increased was not determined but the candidates are P2X2, P2X4 and P2X5Rs, but not P2X7Rs, which were reported to be equally present in SGCs in ganglia from both controls and treated animals ${ }^{31}$. Increased responses to ATP were also observed in a mouse model of systemic inflammatory pain in SGCs of the $\mathrm{DRG}^{27}$ and nodose ganglia $^{61}$. It thus appears that $\mathrm{P} 2 \mathrm{R}$ upregulation is part of the SGC activation after injury. There is little information on the pharmacology of SGCs in non-rodent mammals, but P2X7R expression is reportedly upregulated in DRG SGCs of patients with neuropathic pain ${ }^{70}$. These changes, combined with the increased sensitivity of sensory neurons to ATP under pathological conditions $s^{67,71}$, indicate that increased intercellular communication via P2Rs is likely to contribute to neuronal hyperexcitability in pain states (FIG. 2a,b).

Sensory neurons contain several neuroactive peptides, among which calcitonin gene-related peptide (CGRP) is one of the best studied, as it appears to be a major factor in migraine. In the TG of humans, rats and monkeys, the neurons express CGRP and SGCs express components of the CGRP receptor complex ${ }^{72,73}$, which suggests that this peptide mediates neuron-SGC communication. Recently, antibodies against CGRP and its receptors have been approved for migraine therapy, and it was found that they enter sensory ganglia but not the $\mathrm{CNS}^{74}$. This raises the possibility that the therapeutic action of these antibodies may involve disruption of abnormal neuron-SGC interactions.

Further study of SGC pharmacology is crucial for understanding the function of SGCs because chemical signalling is the main mode of glia-neuron communication. Recent technical developments allow recording of the physiological activity of sensory neurons in intact mice ${ }^{44,75,76}$, and this method can be utilized to explore the pharmacology of SGCs in vivo.

SGCs and intercellular communication. Neurons in sensory ganglia receive no synapses and are separated from each other by SGCs and the connective tissue space ${ }^{4}$ (FIG. 1 c). It would therefore appear that no interactions among neurons in these ganglia are present; however, Devor and Wall ${ }^{77}$ showed that electrical activity in DRG neurons evokes depolarization in adjacent neurons, and named this 'cross depolarization' (FIG. 2c,d). Later work indicated that the effect is due to the release of unidentified chemical mediators ${ }^{78,79}$. Currently, there is evidence that SGCs contribute to cross depolarization, and that they interact chemically among themselves and with neurons via P2Rs (REFS ${ }^{2,5,68,80,81}$ ) and by gap junctions ${ }^{45}$. A calcium imaging study on the DRG in intact mice found that electrical activity in one neuron can activate neighbouring ones ('coupled activation') ${ }^{44}$. This effect was greatly increased after nerve injury or peripheral inflammation, and was attributed to $\mathrm{Cx} 43$ upregulation in SGCs. Coupled activation was reduced by local or systemic application of the gap junction blocker CBX and also in $\mathrm{Cx} 43$ knockout mice. Pain hypersensitivity behaviour was reduced by CBX. These observations indicate that gap junctions in SGCs are a major contributor to coupled activation and pain. The underlying mechanism of coupled activation is not fully clarified, but it was suggested that neuron-SGC-SGC-neuron electrical coupling is involved ${ }^{44}$ (see FIG. 3a,b). This idea is consistent with the enhanced cell coupling observed in rodent pain model ${ }^{3,80}$, but the contribution of chemical interactions was not excluded.

Studies on short-term TG cultures have shown that SGCs can transmit $\mathrm{Ca}^{2+}$ waves, which enable SGCs (together with neurons) to propagate signals over long distances $^{66,67}$ (FIG. 2a,b). These waves are mediated by gap junctions and chemical messengers (ATP and glutamate). After nerve injury, gap junctions are upregulated and the sensitivity of both neurons and SGCs to ATP increases. It is thus expected that neuron-neuron interactions will be increased after injury, as indeed was observed in mouse pain models ${ }^{44}$.

Nerve damage induces prominent changes in SGCs, and as the injury site can be a large distance from the ganglion, determining which neuronal signals induce these changes has been a puzzle. A likely explanation is that the high firing rate in injured neurons causes the release of chemical mediators that act on SGCs and 

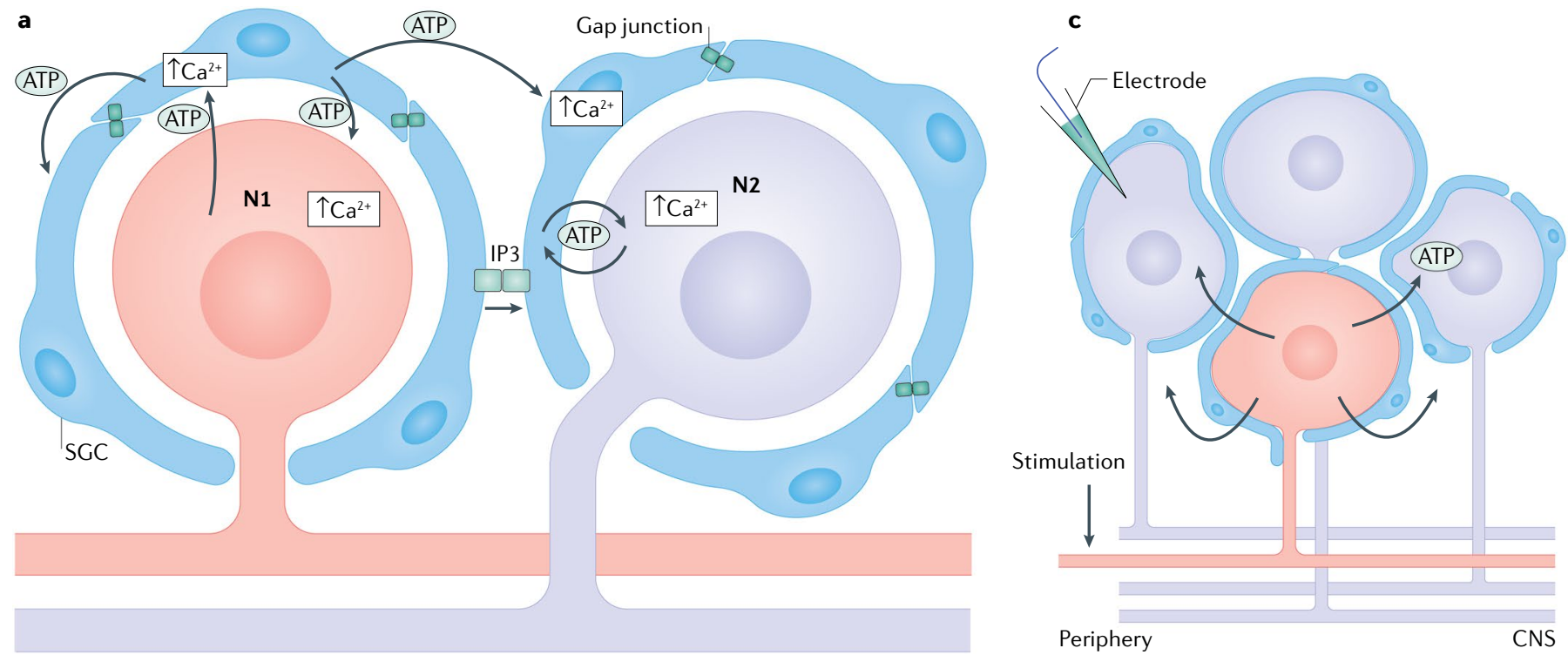

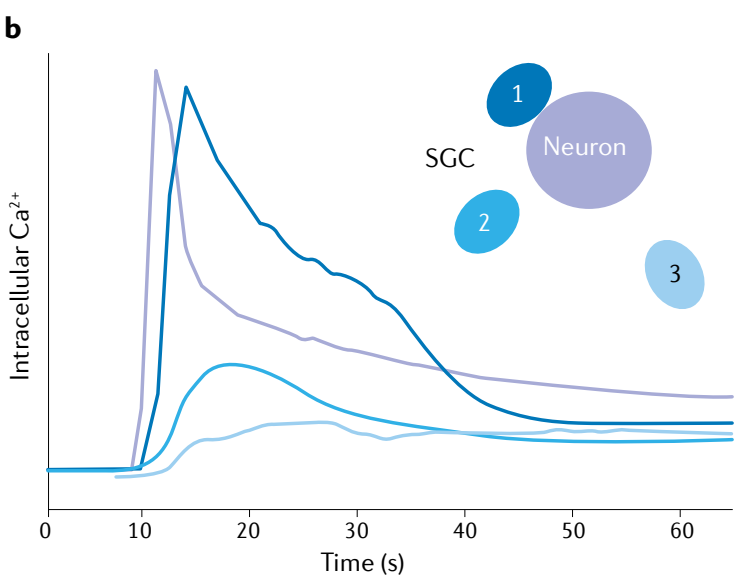

Fig. 2 | Mechanisms of signal spread in sensory ganglia and their possible contribution to chronic pain. a | Spread of calcium waves in sensory ganglia. The axon of neuron 1 (N1) is injured, which induces the firing of action potentials in the cell body. This causes release of ATP from the neuron, which acts on satellite glial cells (SGCs) by elevating intracellular $\mathrm{Ca}^{2+}$ in them, which in turn induces them to release ATP (and other factors, including cytokines). The increased gap junctions between SGCs facilitate the spread of IP3 within the coupled SGCs, which increases the intracellular calcium in these cells. The combination of the increased sensitivity to ATP and the increased coupling by gap junctions will promote the propagation of calcium waves, resulting in the excitation of N2. This excitation may relay nociceptive signals to the spinal cord. $\mathbf{b}$ | Example of calcium wave propagation based on experiments on cultured neurons and SGCs from mouse trigeminal ganglion. Electrical or mechanical stimulation of the neuron at time 0 resulted in higher intracellular $\mathrm{Ca}^{2+}$ in it. After a delay, the adjacent $\mathrm{SGC}$ s also responded with elevated intracellular $\mathrm{Ca}^{2+}$, with the amplitude and delay determined by proximity to the neuron. Similar results

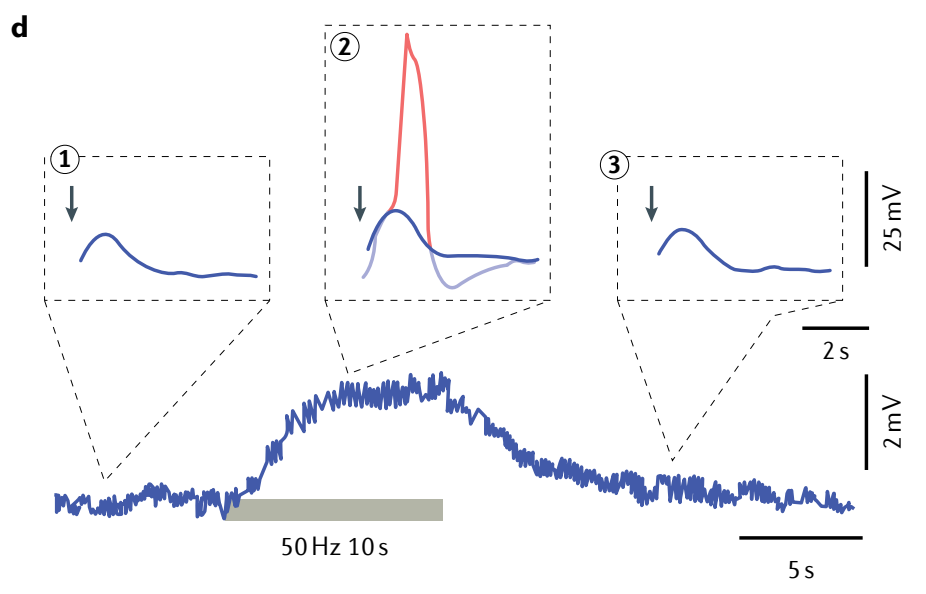

were obtained when an SGC was stimulated. Inset: schematic showing a neuron and three SGCs (labelled 1-3). c,d | Cross depolarization in the DRG. The method for demonstrating cross depolarization: stimulating the axon of a neuron (coloured red) evokes firing in it, and intracellular recording (blue electrode) from an unstimulated neuron reveals slow depolarization due to this firing (part c). Brief subthreshold depolarizing currents (indicated by downward-pointing arrows) were applied to the recorded cell before (1), during (2) and after (3) the stimulation of an axon of a nearby neuron. When applied while the neuron was depolarized by the excitation of the nearby neuron (marked by the bar below the lower trace), the brief electrical stimulus summed with the cross depolarization to reach the threshold and fire an action potential (red trace) (part d). The conclusion from this experiment is that electrical activity in one neuron releases a messenger (likely ATP), causing cross depolarization, which increases the excitability of other neurons. Part a adapted with permission from REF. ${ }^{80}$, Elsevier. Part $\mathbf{b}$ adapted with permission from REF. ${ }^{66}$, Cambridge University Press. Part d adapted from REF. ${ }^{78}$ copyright 1996 , Society for Neuroscience.

\section{Neuralgia}

Pain extending along the course of nerves; for example, trigeminal neuralgia. activate them ${ }^{82}$. Such mediators may include cytokines, growth factors and nitric oxide. Active neurons release nitric oxide, which diffuses rapidly over the narrow gap between them and the SGCs. Nitric oxide mimics the observed changes in SGCs that occur after nerve insult ${ }^{83}$, and thus appears to be a key element in SGC activation.
We have proposed a scheme to explain how increased gap junctional communication and sensitization to ATP can explain the role of SGCs in neuronal hyperexcitability in pain models ${ }^{45}$, based on the 'ignition theory' formulated to account for trigeminal neuralgia ${ }^{84}$. In this model, hyperexcitability of sensory neurons is evoked 
by nerve injury. Activation of one neuron then spreads to others, achieving synchronized and sustained activity within neuronal ensembles. In the original framework, the synchronized discharge was hypothesized to result from chemical cross-excitation. It is conceivable that the increased neuronal activity leads to ATP release through upregulated Panx1 channels and that responses are amplified as a consequence of $\mathrm{P} 2 \mathrm{R}$ activation in SGCs, and additional opening of Panx1 channels. Release of other molecules, including nitric oxide, cytokines and growth factors, likely also contributes to SGC activation. A modified ignition model based on
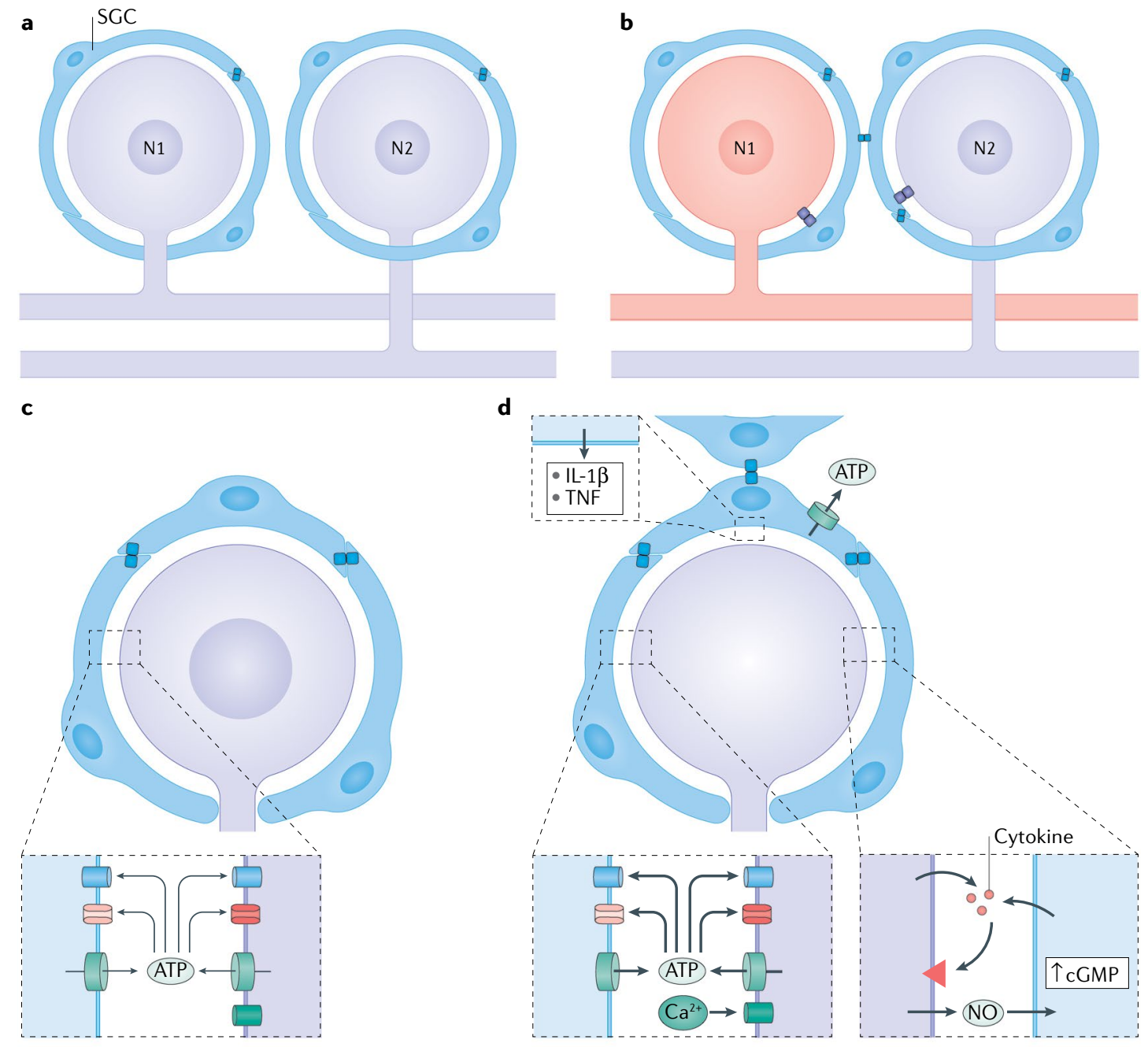

d

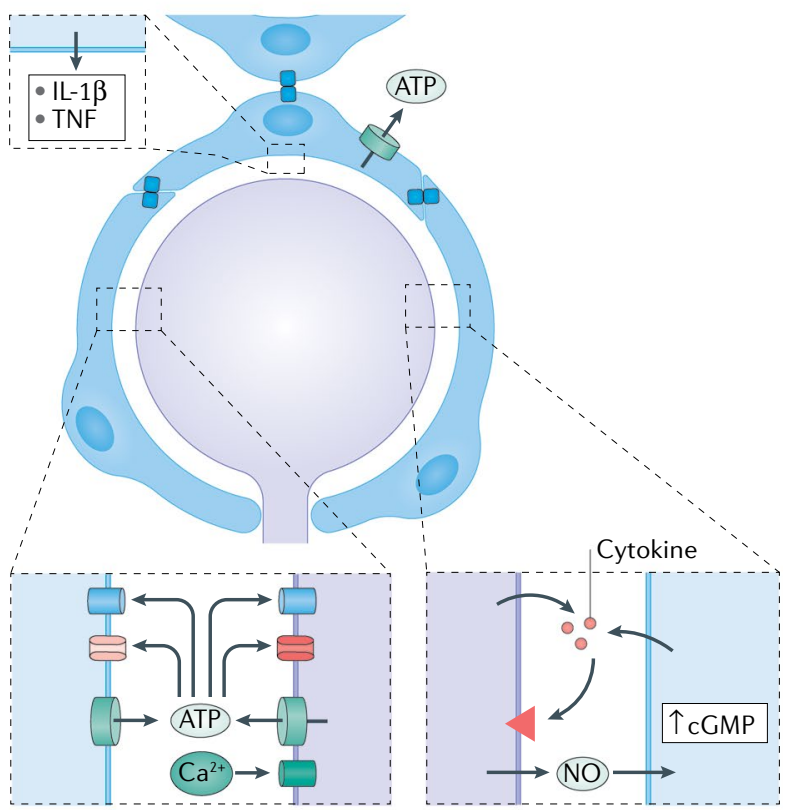

\begin{tabular}{|c|c|c|}
\hline SGC-SGC gap junction & $\mathrm{P} 2 \mathrm{X} 3 \mathrm{R}$ & TRPV1 \\
\hline SGC-neuron gap junction & $\mathrm{P} 2 \mathrm{X} 7 \mathrm{R}$ & Cytokine receptor \\
\hline$\circlearrowleft$ Panx1 & P2YR & \\
\hline
\end{tabular}

Fig. 3 | Injury-induced changes in neuron-SGC bidirectional communications. a,b | Proposed mechanism of coupled activation between neurons. Synchronous activity of adjacent neurons could arise by the spread of depolarization from neuron 1 (N1) to its surrounding satellite glial cells (SGCs), then through gap junctions to SGCs of a nearby neuron and then through gap junctions from these SGCs to N2. Under control conditions, SGCs are coupled mostly to other SGCs around a given neuron (part a). After peripheral injury or inflammation to a neuron (coloured red), SGCs become more strongly coupled to other SGCs around the same neuron (and also to neurons) by newly formed gap junctions. This enables increased transfer of electrical current and small molecules among SGCs and between SGCs and neurons. Such a mechanism can account for coupled neuronal activation (part b). c,d | The chain of events connecting neuronal excitation and glial activation. Resting conditions are shown in part c. Following neuronal damage, the neuronal cell body fires a high rate of action potentials, which increases intracellular calcium that in turn activates nitric oxide synthase to produce nitric oxide. Nitric oxide diffuses from the neuron and reaches the surrounding SGCs, where it induces cyclic guanosine 5'-monophosphate (cGMP) synthesis. This second messenger can have various actions on SGCs, and may be a key factor in SGC activation. SGC activation includes the release of ATP and cytokines, gap junction formation and increased sensitivity to ATP (part d). NO, nitric oxide; P2R, P2 purinergic receptor; Panx1, pannexin 1; TNF, tumour necrosis factor; TRPV1, transient receptor potential vanilloid type 1 channel. 
Lipopolysaccharide

(LPS). A component of the wall

of Gram-negative bacteria;

LPS acts on Toll-like receptor 4

(TLR4), which, in sensory

ganglia, is located on the

surface of the sensory neurons.

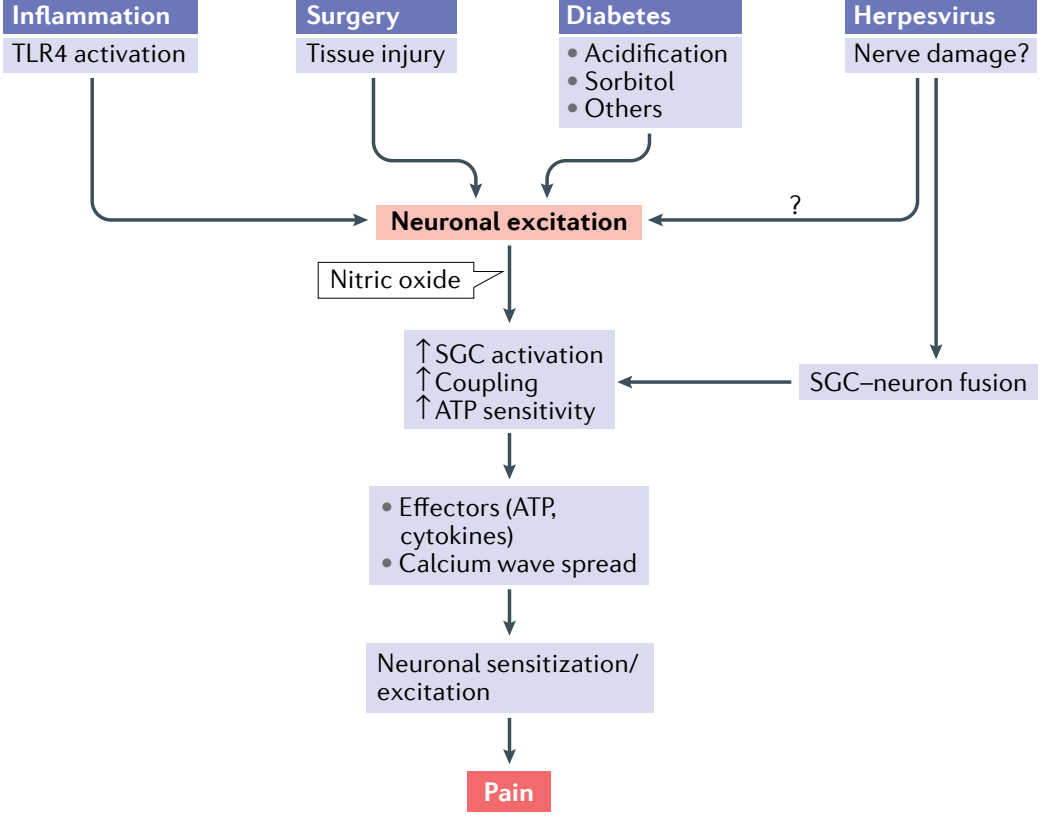

Fig. 4 | Proposed sequence of events connecting nerve injury in four different pain models to SGC activation and neuronal hyperexcitability. This sequence is an attempt to generalize the main events, but does not exclude additional or alternative mechanisms. The initial event in this cascade is injury to sensory neurons, such as from surgery, inflammation, diabetes or herpesvirus, which increases their firing rate. This leads to production of nitric oxide in the neurons. Nitric oxide diffuses from the injured neurons and activates satellite glial cells (SGCs) that surround them and likely also SGCs around adjacent neurons. SGC activation involves numerous processes, including greater coupling by gap junctions, increased sensitivity to ATP, upregulation of ERK and increased release of pro-inflammatory cytokines (IL-1 $\beta$, tumour necrosis factor, IL-6, fractalkine and others). The increased gap junctions and sensitivity to ATP will enhance calcium waves, which lead to neuronal excitation. Cytokines released from SGCs will also contribute to neuronal sensitization and excitation. The overall effect will be greater firing (spontaneous and/or evoked) of sensory neurons and pain. TLR4, Toll-like receptor 4. localized and systemic types of chronic pain (somatic, visceral and orofacial). Several studies have demonstrated that reversing injury-induced changes in SGCs reduced or abolished pain behaviour in rodent models. Below, we describe the possible contributions of SGCs to several pain syndromes that are commonly encountered in clinical practice.

Systemic inflammation. Systemic inflammation is a common human disease. The ensemble of symptoms associated with this disorder is called 'sickness behaviour', and includes depression and pain ${ }^{90}$. As for most other pain syndromes, many investigators have emphasized the role of central mechanisms in sickness behaviour, with increased cytokine levels being a major factor. However, recent work suggests that activity initiated by LPS injection and sustained within the sensory ganglia contributes to the generation and maintenance of pain in systemic inflammation ${ }^{27}$. Moreover, a single intraperitoneal LPS injection induced changes in the DRG, which were associated with mechanical hypersensitivity that lasted for at least a month ${ }^{86}$. Apparently, the direct effects of LPS are short-lived and depend on its binding to Toll-like receptor 4 (TLR4) in sensory neurons, but the downstream sequelae can last for weeks. LPS-induced changes in SGCs were similar to those observed in local inflammatory pain models, which include SGC activation and increased SGC-SGC, neuron-SGC and neuron-neuron dye coupling by gap junctions ${ }^{19,50,51}$. Increased numbers of gap junctions in SGCs were detected by electron microscopy at both 7 and 30 days post LPS administration ${ }^{27,86}$. Neuronal gap junctions were not detected either before or after LPS injection, which is consistent with the very weak coupling of neurons to each other and to SGCs ${ }^{53}$. In addition, LPS administration increased the responses of SGCs to ATP and reduced the withdrawal threshold in response to mechanical stimulation of the paw or abdomen. Intraperitoneal injection of the gap junction blocker CBX or palmitoleic acid raised the pain threshold back to the control level, suggesting a role for gap junctions in pain. These (and other) gap junction blockers are not highly specific and may act by other mechanisms and locations (for example, those that cross the blood-brain barrier may act centrally). However, CBX does not cross the blood-brain barrier ${ }^{91}$ and is therefore likely to act largely in the periphery, and gap junction blockers did not affect the withdrawal threshold in control mice. These findings support the idea that increased gap junction expression in sensory ganglia contributes to chronic pain, and indicate that gap junction blockade in these ganglia has therapeutic potential for alleviating chronic pain.

Chronic post-surgical pain (PSP). PSP can be acute (resolving within several days after the operation) or chronic (lasting months and even years). Chronic PSP is observed in $10-50 \%$ of patients undergoing common operations, such as hip and knee operations and mastectomy ${ }^{92,93}$. Current therapy for chronic PSP is inadequate, and preventive treatments have been largely ineffective ${ }^{94}$.

It is thought that chronic PSP is caused by nerve injury - that is, it is neuropathic - but this topic is 
Central sensitization

A state when the central nervous system becomes highly reactive, causing even mild stimuli to be sensed as painful.

Extracellular-signal regulated kinase (ERK). A member of the MAP kinase family that is involved in multiple cellular processes. controversial $^{92}$. For example, skin and muscle incision and retraction in rats without visible nerve injury induced chronic PSP that persisted for more than 3 weeks ${ }^{95}$, suggesting that mechanisms other than nerve injury are involved in chronic PSP. It is assumed that chronic PSP is associated with central sensitization ${ }^{96}$, but peripheral mechanisms are also important ${ }^{97-99}$, as peripheral activity drives the central sensitization in PSP $^{100}$ and in other pain states ${ }^{101}$.

Little is known regarding peripheral mechanisms in chronic PSP. Activation of P2X7R on SGCs was found to elevate phosphorylated ERK (pERK1/2) in SGCs, which led to TNF release ${ }^{98}$. TNF in turn acts on neurons, which increases neuronal excitability. These results indicate that SGCs are a key element in chronic PSP generation and may be a highly suitable therapeutic target for it. Sensory ganglia are not protected by a vascular barrier, and therefore SGCs, which surround the neurons, are ideal targets for pain therapy.

In a PSP model based on paw incision, $\mathrm{pERK} 1 / 2$ expression was found to be increased 2 -fold in A-fibre neurons and SGCs in the DRG. This increase was observed as early as 2 min after the incision, and returned to baseline at $2 \mathrm{~h}^{99}$. This effect is extremely fast, especially considering that SGCs are not influenced directly by the injury and must receive signals from the neurons. The local anaesthetic levobupivacaine inhibited pERK1/2 induction. CBX, which blocks both gap junctions and Panxl channels, inhibited the early glial pERK1/2 increase and also inhibited pain hypersensitivity ${ }^{99}$. This study indicates that early events induced in SGCs by nerve injury participate in the generation of hypersensitivity in PSP. It is thus likely that preventing these events will have therapeutic value in PSP treatment.

Diabetic neuropathic pain (DNP). Nerve damage (neuropathy) is a common complication in diabetes mellitus type 1 and 2; it affects about $50 \%$ of the patients ${ }^{102}$ and is difficult to treat ${ }^{103}$. A frequent consequence of this neuropathy is DNP. Little is known about the mechanisms responsible for DNP, but it is clear that both central and peripheral mechanisms contribute ${ }^{102,104}$.

The most widely used diabetes mellitus type 1 model is obtained by injecting rodents with the toxin STZ, which destroys pancreatic beta cells. The key element in diabetes mellitus models is a several-fold increase in the blood glucose level, which induces numerous types of cellular damage.

Injury to the peripheral nervous system in diabetes mellitus is well recognized ${ }^{102}$. Research has mostly focused on axons, but there is evidence that functional changes in sensory neurons can contribute to $\mathrm{DNP}^{105-108}$. Ionic currents in sensory neurons are abnormal in diabetes mellitus ${ }^{105-107}$, indicating that sensory ganglia are highly suitable targets for research and therapy of DNP. SGCs can release the cytokines TNF and IL-1 $\beta$, particularly following insults ${ }^{2,36,98}$, so neuronal excitation by SGC-derived cytokines may be one way in which SGCs contribute to DNP.

Altered enzymatic activity in SGCs might be relevant to cellular damage in diabetes mellitus. One such enzyme is aldose reductase ${ }^{109}$, which catalyses the conversion of glucose into sorbitol. Increased sorbitol production in cells leads to its accumulation, causing osmotic swelling and cell damage $\mathrm{e}^{109,110}$. Aldose reductase activity is present in SGCs (but not in neurons) of rat $\mathrm{DRG}^{111}$. Targeting aldose reductase in SGCs seems to have promising potential for treating DNP, and efforts are underway to develop aldose reductase inhibitors as therapy for diabetes mellitus complications ${ }^{112}$.

There is evidence for SGC activation in the STZ mode $^{89}$. The underlying mechanism for this activation appears to be related to the hyperglycaemia-induced upregulation of pyruvate dehydrogenase kinases (PDK2 and PDK4) in DRG cells (SGCs, neurons and infiltrating macrophages). These enzymes play key roles in glucose metabolism ${ }^{105}$, and their enhanced activity causes lactic acidosis, which activates SGCs and macrophages ${ }^{105}$. Reactive SGCs and macrophages release pro-inflammatory cytokines (TNF, IL-1 $\beta$ and IL-6), which increase neuronal excitability and thus lead to pain. It can be concluded from this work ${ }^{105}$ that PDKs play a pivotal role in inducing SGC activation and creating a pro-inflammatory microenvironment in diabetic DRG, the prerequisites for pain pathogenesis.

The pharmacology of SGCs in diabetes mellitus models has received only little attention. It was found that in parallel with the activation of SGCs in the STZ model, the sensitivity of these cells to the P2X7R agonist BzATP was increased ${ }^{113}$. However, BzATP is a potent agonist of other P2XRs (REF. ${ }^{114}$ ), particularly P2X4R, and indeed there is evidence that $\mathrm{P} 2 \mathrm{X} 4 \mathrm{R}$ is upregulated in SGCs in the STZ model and that it is essential for the mechanical hypersensitivity observed in this model ${ }^{115}$. This topic needs to be further explored, particularly from the perspective of the degree to which SGC-neuron interaction is compromised and whether $\mathrm{Cx} 43$ or Panx1 is involved in the heightened P2R sensitivity in SGCs.

Post-herpetic neuralgia. Alphaherpesviruses, varicella zoster virus (VZV), herpes simplex virus type 1 (HSV-1) and HSV-2 infect the peripheral nervous system. After the primary infection, these viruses can become latent within sensory ganglia for the lifetime of the host, but retain the ability to reactivate and cause disease episodes. Reactivation of VZV results in herpes zoster, which is characterized by painful skin eruptions (shingles) and often leads to post-herpetic neuralgia ${ }^{116,117}$. Reactivation of HSV causes mucocutaneous lesions (for example, cold sores) and may also result in neuralgia ${ }^{118}$. Post-herpetic neuralgia can be severe, persistent and refractory to treatment $^{116,119}$. The mechanisms underlying this condition are not known. VZV is difficult to study because it does not infect rodents, and instead HSV-1 is used in rodent studies.

The role of SGCs in herpetic infection and pain has been controversial ${ }^{1}$. Major questions are whether SGCs are actually infected and whether they contain the virus during latency. Another open question is whether abnormal SGC-neuronal interactions are present in herpes-infected ganglia, which could potentially contribute to post-herpetic pain.

To overcome the lack of a rodent model for VZV, Zerboni et al. ${ }^{120}$ grafted fetal human DRG under the kidney capsule of severe combined immunodeficiency mice. The grafted ganglia were infected with VZV, and 
examined histologically after various periods (up to 20 weeks). This innovative approach clearly showed that the virus was present in both DRG neurons and SGCs ${ }^{121}$, see BOX 3.

Cutaneous HSV-1 infection in mice was reported to promote infiltration of leukocytes into the DRG, where they release cytokines that downregulate the expression of Kir4.1 channels in SGCs ${ }^{122}$. Although it was assumed that the inflammatory mediators were present in the skin, the authors did not evaluate whether release could be from DRG-resident cells (SGCs, for example). It should also be added that infection of DRG cells by the virus was not verified, and it could be argued that the changes in the DRG could be due to the skin lesion caused by the virus and subsequent local release of cytokines. It is notable that there is no conclusive demonstration of GFAP upregulation in SGCs in herpes, although there is evidence from western blots for GFAP elevation in the DRG after infection with HSV-1 (REF. ${ }^{122}$ ).

Few studies have examined the functional consequences of HSV infection on cells within sensory ganglia. In one such study, freshly dissociated TG neurons and SGCs were infected with HSV-1 and studied after $48 \mathrm{~h}$ in vitro ${ }^{67}$, at which time both the neurons and SGCs were infected. Calcium imaging, dye injections and intracellular electrical recordings from the neurons revealed several notable changes in these cultures. First, intercellular $\mathrm{Ca}^{2+}$ waves, which involved both neurons and SGCs, were more extensive in the infected cultures than in controls, which is consistent with greater spread of neural activity $^{80}$. These waves were largely mediated by ATP acting on P2Rs in neurons and SGCs. Neuronal, but not glial, sensitivity to ATP was increased by the viral infection. Second, dye coupling among cells (neuron-neuron, neuron-SGC and SGC-SGC) was greatly increased. This coupling was not mediated by gap junctions but by cell fusion, which is known to occur following infection with alphaherpesviruses ${ }^{123,124}$, see BOX 3. Apparently, the ATP-mediated $\mathrm{Ca}^{2+}$ wave propagation is facilitated by the direct connection of the cytoplasm of cells. Third, electrical recordings from the neurons showed a widening of the action potentials in neurons from infected cultures compared with controls, consistent with greater influx of $\mathrm{Ca}^{2+}$. This would raise intracellular $\mathrm{Ca}^{2+}$ levels, leading to increased release of mediators, including ATP. Thus, the enhanced $\mathrm{Ca}^{2+}$ waves are explained by greater sensitivity of neurons to ATP, by cell fusion and by greater $\mathrm{Ca}^{2+}$ influx into the neurons. The increased $\mathrm{Ca}^{2+}$ waves and neuronal hyperexcitability may lead to increased neuronal firing, and thus to pain. These results help in understanding the peripheral mechanisms of pain in herpes, but need to be validated in animal models where HSV-1 infection is made in vivo. A limitation of the in vitro work is that it does not provide information on long-term events and reactivation.

\section{SGCs in other types of viral infections}

Infections by viruses other than herpesvirus may also involve SGCs. Of special interest is HIV-1, the virus that causes AIDS, in which peripheral neuropathy and pain are common. Infection with simian immunodeficiency virus is a useful model to study HIV. In macaques

\footnotetext{
Box 3 | SGCs in human disease

Information on satellite glial cells (SGCs) in humans is scarce; however, changes in SGCs have been observed in certain disorders, including herpesvirus infection, AIDS, Friedreich ataxia and herpesvirus infection. In patients infected with herpes (including herpes simplex virus and varicella zoster virus (VZV)), the virus resides in sensory ganglia for life, but the role of SGCs in herpetic infection and pain has been controversial. Zerboni et al. ${ }^{120}$ grafted human embryonic dorsal root ganglia (DRG) in mice (see main text), and established that SGCs are infected by VZV before the neurons are. SGCs from the DRG of patients with Friedreich ataxia form abnormal onion-like layers around the neurons ${ }^{121}$ (see the figure, part a), where SGCs are coloured green (for the VZV protein IE63), and surround a neuron (N1), coloured red for RT97, a marker for mechanosensitive neurons; cell nuclei are coloured blue. A similar observation was made in a rat pain model ${ }^{155}$. Herpesvirus infection can cause cell fusion, a process by which cells connect and form a single, larger cell. This facilitates the passage of metabolites as well as the virus between neurons and SGCs ${ }^{123}$ (see the figure, part b). In the case of haemagglutinating encephalomyelitis, which is a member of the coronavirus family (see text), the virus passes between neurons and SGCs by exocytosis from neurons, followed by endocytosis by SGCs (see the figure, part c). Over half of patients with AIDS develop peripheral neuropathy, which is associated with sensory disorders, including pain. In sensory ganglia of these patients it is common to find Nageotte

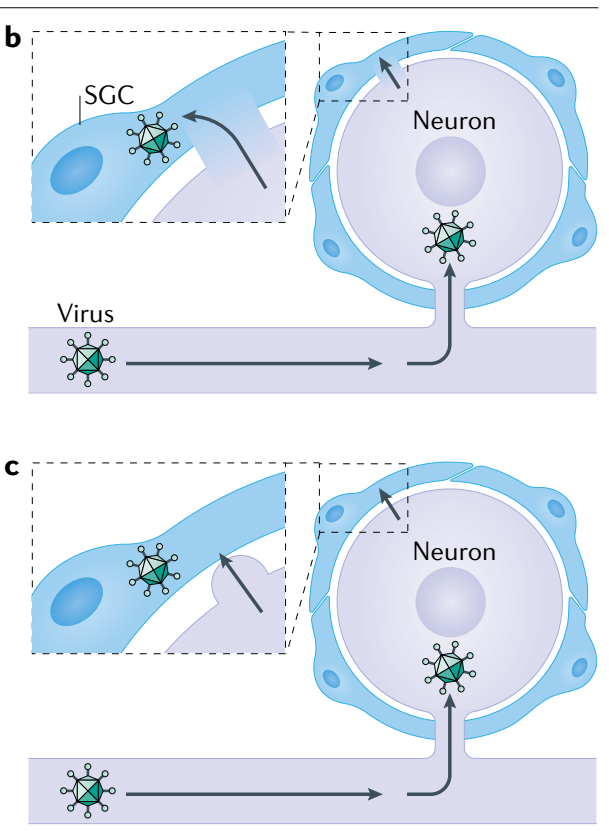

nodules, which are clusters of SGCs that occupy the space of degenerated neurons ${ }^{156}$. Friedreich ataxia is a genetic disease characterized by deficiency in frataxin, a mitochondrial protein. Patients with Friedreich ataxia suffer from impaired movement and cardiac functions. In the DRG of patients with Friedreich ataxia, SGCs are activated and form numerous gap junctions and abnormal multiple layers around the neurons ${ }^{157}$. Part $\mathbf{a}$ adapted from REF. ${ }^{121}$, CC BY 4.0.
} 


\section{DREADD}

(Designer receptors exclusively activated by designer drugs).

A method that utilizes

$\mathrm{G}$ protein-coupled receptors engineered to respond

exclusively to synthetic ligands. infected with simian immunodeficiency virus there was an upregulation of GFAP in DRG SGCs ${ }^{125}$, but its contribution to the disease is not known. Another virus family is coronavirus that includes MERS-CoV, SARS-CoV and SARS-CoV-2 (which causes COVID-19), which are highly infective and can be fatal in humans. Information on the involvement of SGCs in coronavirus infections is minimal, but there are some clues from a study on another member of this family - swine haemagglutinating encephalomyelitis virus, which is highly lethal in pigs. An electron-microscopic study of haemagglutinating encephalomyelitis virus-infected rats found that the virus replicated in DRG neurons ${ }^{126}$. Viral particles were secreted from neurons, and were taken up by SGCs and located in lysosome-like structures, indicating that SGCs can restrict the virus spread. This protective function is consistent with the ability of SGCs to phagocytose pathogens, as described in BOX 1.

\section{SGCs in sympathetic ganglia}

Most of the available knowledge on SGCs in autonomic ganglia is on sympathetic ganglia, and therefore only these ganglia are discussed here. As in sensory ganglia, SGCs in sympathetic ganglia wrap around the neurons, but gaps in the envelope may occur ${ }^{127}$. A major difference between SGCs in sympathetic and sensory ganglia is the presence of synapses in sympathetic ganglia, where SGCs form a layer over the synapses, enabling them to control synaptic transmission ${ }^{127}$ (FIG. 1e). This arrangement is similar to the 'synaptic cradle' proposed for astrocyteneuron arrangement in the $\mathrm{CNS}^{128}$. Acetylcholine (ACh) is the major neurotransmitter in sympathetic ganglia, and indeed it was found that SGCs in the mouse superior cervical ganglion (sympathetic) are sensitive to $\mathrm{ACh}^{129}$. This raises the possibility that SGCs influence synaptic transmission in sympathetic ganglia. SGCs in sensory ganglia are not sensitive to $\mathrm{ACh}^{129}$, which is consistent with the idea that there is a match between chemical messengers in neurons and the receptors in adjacent glia. A later work showed that selective injury to sympathetic nerve terminals activated SGCs in superior cervical ganglia, but not in sensory ones ${ }^{130}$. Conversely, LPS-induced inflammation, which activated SGCs in sensory ganglia, had no effect on SGCs in the superior cervical ganglia ${ }^{130}$. This selective activation correlates with the presence of TLR4 in sensory neurons, and their absence in sympathetic ones. The results above are supported by a recent study ${ }^{131}$, which showed that SGCs in sympathetic ganglia release factors that augment cholinergic synaptic transmission. SGCs also promote synapse formation and contribute to neuronal survival ${ }^{131}$. Thus, SGCs play crucial roles in both development and maintenance of sympathetic function.

There is evidence that the sympathetic nervous system is involved in pain mechanisms, but this topic is controversial. Most notable is the possible role of sympathetic nerves in complex regional pain syndrome ${ }^{132}$. Animal studies have shown that nerve damage augments interactions between sympathetic and sensory ganglia. For example, damage causes sympathetic fibres to sprout in the $\mathrm{DRG}^{133}$, and in two models of DRG nerve injury there was an inflammatory response in sympathetic ganglia (infiltration of immune cells) and SGC activation ${ }^{134}$. These observations suggest that interactions between sensory and sympathetic ganglia may underlie sympathetic contribution to pain.

Sympathetic ganglia play a major role in controlling cardiovascular functions, and in a study where SGCs in mouse superior cervical ganglion were stimulated selectively using DREADD, activation of SGCs increased the heart rate and contractility ${ }^{135}$. It appears that SGC stimulation activated sympathetic fibres that innervate cardiac muscle. This is the first demonstration that SGCs can activate sympathetic nerves, and raises the possibility that sympathetic overactivity, which is a contributing factor in heart failure ${ }^{136}$, is related to abnormal SGC function. These results present a novel approach to better understanding of heart disease and may offer completely novel strategies for therapy.

\section{Future perspectives}

We expect that the increasing interest in SGCs and the application of newly developed research tools will enable progress in novel areas of this field. Future directions in SGC research that we believe may be particularly fruitful are the following. First, injury to sensory neurons causes the attraction of macrophages into the ganglia, and they even penetrate into the space between SGCs and neurons ${ }^{137}$. Macrophages release various pro-inflammatory substances, which can increase neuronal firing ${ }^{138,139}$. Studying macrophage-SGC interactions seems a promising direction. It was found that SGCs and macrophages promote regeneration of DRG axons ${ }^{140}$, and this topic needs to be probed in depth. Second, methods are now available to isolate SGCs, which allows the analysis of their transcriptomes under normal and pathological conditions ${ }^{141}$. For example, single-cell RNA sequencing will enable a search for SGC subtypes and a comparison between SGCs from different types of ganglia, topics on which we currently know nothing. Third, most available information on SGCs has been obtained in rodents, which limits its applicability to clinical situations. Human sensory ganglia are becoming available for laboratory research ${ }^{142}$, which opens exciting possibilities for validating in human material the concepts that have been developed in rodent studies. Using ganglia from patients will help in learning more about the role of SGCs in human disorders, such as migraine and post-herpetic pain. Extending the study on SGCs in viral diseases should receive high priority, as these cells may be a novel therapeutic target. Fourth, use of genetically encoded activity indicators in intact animals potentially allows the observation of interactions between neurons and SGCs in sensory ganglia ${ }^{44,75,76}$ and could provide novel information regarding such interactions in autonomic ganglia as well. These techniques are certain to provide insight into pathological activity patterns and to enable testing of pharmacological strategies to reverse the pathological changes. Finally, current knowledge of SGCs in autonomic ganglia is very limited, but in view of the recent work, which revealed novel and important roles of these cells in sympathetic ganglia, we expect that this area will attract growing attention. 


\section{Concluding remarks}

We describe here possible roles of SGCs in four important pain syndromes. These syndromes have different clinical manifestations, and each may be mediated by different biochemical and physiological mechanisms. Still, the observations that SGCs in sensory ganglia are altered in a similar way in these models (as well as in other models that were not mentioned) suggest that changes in these cells are a general feature of chronic pain. The location of sensory ganglia outside the blood-brain barrier, and the arrangement of SGCs around the neurons, make SGCs an ideal therapeutic target for chronic pain. Similarly, autonomic ganglia are more accessible to intravascular agents than is the brain parenchyma ${ }^{74}$, and SGC modulation of sympathetic output might be targeted for disturbances of heart rhythm, blood pressure and other disorders.

Published online 22 July 2020
1. Hanani, M. Satellite glial cells in sensory ganglia: from form to function. Brain Res. Brain Res. Rev. 48 457-476 (2005)

2. Huang, L. Y., Gu, Y. \& Chen, Y. Communication between neuronal somata and satellite glial cells in sensory ganglia. Glia 61, 1571-1581 (2013)

3. Jasmin, L., Vit, J. P., Bhargava, A. \& Ohara, P. T Can satellite glial cells be therapeutic targets for pain control? Neuron Glia Biol. 6, 63-71 (2010).

4. Pannese, E. Biology and pathology of perineuronal satellite cells in sensory ganglia. Adv. Anat. Embryol. Cell Biol. 226, 1-63 (2018)

5. Rozanski, G. M., Li, Q. \& Stanley, E. F. Transglial transmission at the dorsal root ganglion sandwich synapse: glial cell to postsynaptic neuron communication. Eur. J. Neurosci. 237, 1221-1228 (2013).

6. Bushong, E. A., Martone, M. E., Jones, Y. Z. \& Ellisman, M. H. Protoplasmic astrocytes in CA1 stratum radiatum occupy separate anatomical domains. Neuroscience 22, 183-192 (2002).

7. Ji, R. R., Donnelly, C. R. \& Nedergaard, M. Astrocytes in chronic pain and itch. Nat. Rev. Neurosci. 20, 667-685 (2019)

8. Liddelow, S. A. \& Barres, B. A. Reactive astrocytes: production, function, and therapeutic potential. Immunity 46, 957-967 (2017)

9. Verkhratsky, A. \& Nedergaard, M. Physiology of astroglia. Physiol. Rev. 98, 239-389 (2018).

10. Burnstock, G. Physiology and pathophysiology of purinergic neurotransmission. Physiol. Rev. 87 659-797 (2007)

11. Fields, R. D. \& Burnstock, G. Purinergic signalling in neuron-glia interactions. Nat. Rev. Neurosci. 7 423-436 (2006)

12. Kettenmann, $\mathrm{H}$ \& Zorec, R in Neuroglia 4th edn. (eds Kettenmann, H. \& Ransom, B. R.) 197-211 (Oxford Univ. Press, 2013).

13. Watkins, L. R. \& Maier, S. F. GLIA: a novel drug discovery target for clinical pain. Nat. Rev. Drug. Discov. 2, 973-985 (2003).

14. Grace, P. M., Hutchinson, M. R., Maier, S. F. \& Watkins, L. R. Pathological pain and the neuroimmune interface. Nat. Rev. Immunol. 14, 217-231 (2014).

15. Inoue, K. \& Tsuda, M. Microglia in neuropathic pain cellular and molecular mechanisms and therapeutic potential. Nat. Rev. Neurosci. 19, 138-152 (2018)

16. Chen, G., Zhang, Y. Q., Qadri, Y. J., Serhan, C. N. \& Ji, R. R. Microglia in pain: detrimental and protective roles in pathogenesis and resolution of pain. Neuron 100, 1292-1311 (2018).

17. Scadding, J. W. \& Koltzenburg, M. in Wall and Melzack's Textbook of Pain 6th edn (eds McMahon, S. B. Koltzenburg, M. I., Tracey, I. D. \& Turk, D.) 926-951 (Elsevier Churchill Livingstone, 2013).

18. Devor, M. in Wall and Melzack's Textbook of Pain 6th edn (eds McMahon, S. B., Koltzenburg, M. I., Tracey, I. D. \& Turk, D.) 867-888 (Elsevier Churchill Livingstone, 2013).

19. Huang, T. Y., Belzer, V. $\&$ Hanani, M. Gap junctions in dorsal root ganglia: possible contribution to visceral pain. Eur J. Pain 14, 49 e1-49 e11 (2010).

20. Guha, D. \& Shamji, M. F. The dorsal root ganglion in the pathogenesis of chronic neuropathic pain. Neurosurgery 63, 118-126 (2016).

21. Vaso, A. et al. Peripheral nervous system origin of phantom limb pain. Pain 155, 1384-1391 (2014).

22. Stephenson, J. L. \& Byers, M. R. GFAP immunoreactivity in trigeminal ganglion satellite cells after tooth injury in rats. Exp. Neurol. 131, 11-22 (1995).

23. Warwick, R. A. \& Hanani, M. The contribution of satellite glial cells to chemotherapy-induced neuropathic pain. Eur. J. Pain. 17, 571-580 (2013).

24. Woodham, P., Anderson, P. N., Nadim, W. \& Turmaine, M. Satellite cells surrounding axotomized rat dorsal root ganglion cells increase expression of GFAP-like protein. Neurosci. Lett. 98, 8-12 (1989).

25. Hanani, M., Huang, T. Y., Cherkas, P. S., Ledda, M. $\&$ Pannese, E. Glial cell plasticity in sensory ganglia induced by nerve damage. Neuroscience 114 , 279-283 (2002)

26. Ohara, P. T., Vit, J. P., Bhargava, A. \& Jasmin, L Evidence for a role of connexin 43 in trigeminal pain using RNA interference in vivo. J. Neurophysiol. 100 , 3064-3073 (2008).

This paper shows that $\mathrm{Cx}_{4} 3$ expression increases in SGCs in a pain model, and that blocking this expression reduces pain behaviour.

27. Blum, E., Procacci, P., Conte, V. \& Hanani, M. Systemic inflammation alters satellite glial cell function and structure. A possible contribution to pain. Neuroscience 274, 209-217 (2014).

28. Vit, J. P., Ohara, P. T., Bhargava, A., Kelley, K. \& Jasmin, L. Silencing the Kir4.1 potassium channe subunit in satellite glial cells of the rat trigeminal ganglion results in pain-like behavior in the absence of nerve injury. J. Neurosci. 28, 4161-4171 (2008)

29. Takeda, M., Takahashi, M., Nasu, M. \& Matsumoto, S. Peripheral inflammation suppresses inward rectifying potassium currents of satellite glial cells in the trigeminal ganglia. Pain 152, 2147-2156 (2011)

30. Tang, X., Schmidt, T. M., Perez-Leighton, C. E. \& Kofuji, P. Inwardly rectifying potassium channel Kir4.1 is responsible for the native inward potassium conductance of satellite glial cells in sensory ganglia. Neuroscience 166, 397-407 (2010).

Together with Vit et al. (2008) and Takeda et al. (2011), this study reports that $\mathrm{K}^{+}$channel Kir4.1 expression increases in SGCs following nerve damage, which can lead to neuronal hyperexcitability.

31. Kushnir, R., Cherkas, P. S. \& Hanani, M. Peripheral inflammation upregulates $\mathrm{P} 2 \mathrm{X}$ receptor expression in satellite glial cells of mouse trigeminal ganglia: a calcium imaging study. Neuropharmacology 61 739-746 (2011).

This paper describes an increased sensitivity of SGCs to ATP in two pain models in mice.

32. Dubovȳ, P., Klusákovă, I., Svízenská, I. ¿ Brázda, V. Satellite glial cells express IL-6 and corresponding signal-transducing receptors in the dorsal root ganglia of rat neuropathic pain model. Neuron Glia Biol. 6 , 73-83 (2010)

33. Souza, G. R. et al. Fractalkine mediates inflammatory pain through activation of satellite glial cells. Proc. Natl Acad. Sci. USA 110, 11193-11198 (2013).

34. Afroz, S. et al. CGRP induces differential regulation of cytokines from satellite glial cells in trigeminal ganglia and orofacial nociception. Int. J. Mol. Sci. 20, 711 (2019).

35. Mitterreiter, J. G. et al. Satellite glial cells in human trigeminal ganglia have a broad expression of functional Toll-like receptors. Eur. J. Immunol. 47, 1181-1187 (2017)

36. Takeda, M., Takahashi, M. \& Matsumoto, S. Contribution of the activation of satellite glia in sensory ganglia to pathological pain. Neurosci. Biobehav. Rev. 33, 784-792 (2009).

37. Cherkas, P. S. et al. The effects of axotomy on neurons and satellite glial cells in mouse trigeminal ganglion. Pain 110, 290-298 (2004).

38. Cui, Y. et al. Astroglial Kir4.1 in the lateral habenula drives neuronal bursts in depression. Nature 554 323-327 (2018)

39. Manteniotis, S. et al. Comprehensive RNA-Seq expression analysis of sensory ganglia with a focus on ion channels and GPCRs in trigeminal ganglia. PLOS ONE 8, e79523 (2013).

40. Procacci, P., Magnaghi, V. \& Pannese, E. Perineuronal satellite cells in mouse spinal ganglia express the gap junction protein connexin 43 throughout life with decline in old age. Brain Res. Bull. 75, 562-569 (2008).

41. Lee-Kubli, C. A. et al. Analysis of the behavioral, cellula and molecular characteristics of pain in severe rodent spinal cord injury. Exp. Neurol. 278, 91-104 (2016).

42. Kaji, K. et al. Connexin 43 contributes to ectopic orofacial pain following inferior alveolar nerve injury. Mol. Pain. 12, 1-12 (2016)

43. Komiya, H. et al. Connexin 43 expression in satellite glial cells contributes to ectopic tooth-pulp pain. J. Oral. Sci. 60, 493-499 (2018).

44. Kim, Y. S. et al. Coupled activation of primary sensory neurons contributes to chronic pain. Neuron 91 , 1085-1096 (2016).

This first study of calcium imaging in sensory neurons in live mice describes the role of the SGC gap junctions in increasing neuronal interactions and pain.

45. Spray, D. C. \& Hanani, M. Gap junctions, pannexins and pain. Neurosci. Lett. 695, 46-52 (2019).

46. Garrett, F. G. \& Durham, P. L. Differential expression of connexins in trigeminal ganglion neurons and satellite glial cells in response to chronic or acute joint inflammation. Neuron Glia Biol. 4, 295-306 (2008).

47. Pannese, E., Ledda, M., Cherkas, P. S., Huang, T. Y. $\&$ Hanani, M. Satellite cell reactions to axon injury of sensory ganglion neurons: increase in number of gap junctions and formation of bridges connecting previously separate perineuronal sheaths. Anat. Embryol. 206, 337-347 (2003).

48. Huang, T. Y. \& Hanani, M. Morphological and electrophysiological changes in mouse dorsal root ganglia after partial colonic obstruction. Am. J. Physiol. Gastrointest. Liver Physiol. 289, G670-G678 (2005)

49. Song, D. D., Li, Y., Tang, D., Huang, L. Y. \& Yuan, Y. Z. Neuron-glial communication mediated by TNF- $\alpha$ and glial activation in dorsal root ganglia in visceral inflammatory hypersensitivity. Am. J. Physiol. Gastrointest. Liver Physiol. 306, G788-G795 (2014).

50. Dublin, P. \& Hanani, M. Satellite glial cells in sensory ganglia: their possible contribution to inflammatory pain. Brain Behav. Immun. 21, 592-598 (2007). This paper provides evidence that blocking the increased SGC coupling can reduce pain behaviour in mice.

51. Ledda, M., Blum, E., De Palo, S. \& Hanani, M. Augmentation in gap junction-mediated cell coupling in dorsal root ganglia following sciatic nerve neuritis in the mouse. Neuroscience 164, 1538-1545 (2009).

52. Jin, Y. Z. et al. Connexin 43 contributes to temporomandibular joint inflammation inducedhypernociception via sodium channel 1.7 in trigemina ganglion. Neurosci. Lett. 707, 134301 (2019).

53. Spray, D. C. et al. Gap junction mediated signaling between satellite glia and neurons in trigeminal ganglia. Glia 67, 791-801 (2019). This study uses dual whole-cell voltage clamp methodology to quantify gap junction-mediated coupling between SGCs and neurons in dissociated trigeminal ganglion cultures.

54. Durham, P. L. \& Garrett, F. G. Neurological mechanisms of migraine: potential of the gap-junction modulator tonabersat in prevention of migraine. Cephalalgia 29, 1-6 (2009).

55. Hanstein, R. et al. Focal inflammation causes carbenoxolone-sensitive tactile hypersensitivity in mice. Open. Pain. J. 3, 123-133 (2010).

56. Lemes, J. B. P. et al. Participation of satellite glial cells of the dorsal root ganglia in acute nociception. Neurosci. Lett. 676, 8-12 (2018).

57. Sosinsky, G. et al. Pannexin channels are not gap junction hemichannels. Channels 5, 193-197 (2011).

58. Dahl, G., Qiu, F. \& Wang, J. The bizarre pharmacology of the ATP release channel pannexin 1 . Neuropharmacology 75, 583-593 (2013). 
59. Zhang, Y., Laumet, G., Chen, S. R., Hittelman, W. N. \& Pan, H. L. Pannexin-1 up-regulation in the dorsal root ganglion contributes to neuropathic pain development. J. Biol. Chem. 290, 14647-14655 (2015).

60. Hanstein, R., Hanani, M., Scemes, E. \& Spray, D. C. Glial pannexin 1 contributes to tactile hypersensitivity in a mouse model of orofacial pain. Sci. Rep. 6, 38266 (2016).

61. Feldman-Goriachnik, R., Belzer, V. \& Hanani, M Systemic inflammation activates satellite glial cells in the mouse nodose ganglion and alters their functions. Glia 63, 2121-2132 (2015)

62. Magni, G., Riccio, D. \& Ceruti, S. Tackling chronic pain and inflammation through the purinergic system. Curr. Med. Chem. 25, 3830-3865 (2018).

This paper summarizes purinergic interactions between neurons and glia, with the emphasis on sensory ganglia.

63. Braun, N. et al. Association of the ecto-ATPase NTPDase 2 with glial cells of the peripheral nervous system. Glia 45, 124-132 (2004).

64. Weick, $\mathrm{M}$. et al. $\mathrm{P} 2$ receptors in satellite glial cells in trigeminal ganglia of mice. Neuroscience 120 , 969-977 (2003)

65. Ceruti, S., Fumagalli, M., Villa, G., Verderio, C. \& Abbracchio, M. P. Purinoceptor-mediated calcium signaling in primary neuron-glia trigeminal cultures. Cell Calcium 43, 576-590 (2008).

66. Suadicani, S. O. et al. Bidirectional calcium signaling between satellite glial cells and neurons in cultured mouse trigeminal ganglia. Neuron Glia Biol. 6, 43-51 (2010).

67. Warwick, R. A. \& Hanani, M. Involvement of aberrant calcium signalling in herpetic neuralgia. Exp. Neurol. 277, 10-18 (2016)

68. Zhang, X., Chen, Y., Wang, C. \& Huang, L. Y. Neuronal somatic ATP release triggers neuron-satellite glial cell communication in dorsal root ganglia. Proc. Nat Acad. Sci. USA 104, 9864-9869 (2007). This is the first report on the release of ATP from sensory neurons, which acts on $\mathrm{P} 2 \mathrm{X} 7$ receptors in SGCs and induces them to release TNF, which in turn increases neuronal excitability.

69. Xu, G. Y., Shenoy, M., Winston, J. H., Mittal, S. \& Pasricha, P. J. P2X receptor-mediated visceral hyperalgesia in a rat model of chronic visceral hypersensitivity. Gut 57, 1230-1237 (2008).

70. Chessell, I. P. et al. Disruption of the P2X7 purinoceptor gene abolishes chronic inflammatory and neuropathic pain. Pain 114, 386-396 (2005).

71. Zhou, J., Chung, K. \& Chung, J. M. Development of purinergic sensitivity in sensory neurons after peripheral nerve injury in the rat. Brain Res. 915 161-169 (2001)

72. Eftekhari, S. et al. Differential distribution of calcitonin gene-related peptide and its receptor components in the human trigeminal ganglion. Neuroscience 169, 683-696 (2010)

73. Eftekhari, S. et al. Localization of CGRP, CGRP receptor, PACAP and glutamate in trigeminal ganglion. Relation to the blood-brain barrier. Brain Res. 1600 93-109 (2015).

74. Noseda, R. et al. Fluorescently-labeled fremanezumab is distributed to sensory and autonomic ganglia and the dura but not to the brain of rats with uncompromised blood brain barrier. Cephalalgia $\mathbf{4 0}$, 229-240 (2020).

75. Chen, C. et al. Long-term imaging of dorsal root ganglia in awake behaving mice. Nat. Commun. 10, 3087 (2019)

76. Emery, E. C. et al. In vivo characterization of distinct modality-specific subsets of somatosensory neurons using GCaMP. Sci. Adv. 2, 11 (2016)

77. Devor, M. \& Wall, P. D. Cross-excitation in dorsal root ganglia of nerve-injured and intact rats. J. Neurophysiol. 64, 1733-1746 (1990).

78. Amir, R. \& Devor, M. Chemically mediated crossexcitation in rat dorsal root ganglia. J. Neurosci. 16, 4733-4741 (1996)

79. Oh, E. J. \& Weinreich, D. Chemical communication between vagal afferent somata in nodose ganglia of the rat and the guinea pig in vitro. J. Neurophysiol. 87, 2801-2807 (2002).

Together with Amir and Devor (1996), this study presents electrophysiological evidence that cross depolarization is chemically mediated

80. Hanani, M. Intercellular communication in sensory ganglia by purinergic receptors and gap junctions: implications for chronic pain. Brain Res. 1487 183-191 (2012)

81. Carvalho, G. B., Mulpuri, Y., Damasio, A. \& Spigelman, I. A role for the $\mathrm{P} 2 \mathrm{Y} 1$ receptor in nonsynaptic cross-depolarization in the rat dorsal root ganglia. Neuroscience 423, 98-108 (2019).

82. Xie, W., Strong, J. A. \& Zhang, J. M. Early blockade of injured primary sensory afferents reduces glial cell activation in two rat neuropathic pain models. Neuroscience 160, 847-857 (2009).

83. Belzer, V. \& Hanani, M. Nitric oxide as a messenger between neurons and satellite glial cells in dorsal root ganglia. Glia 67, 1296-1307 (2019).

84. Devor, M., Amir, R. \& Rappaport, Z. H. Pathophysiology of trigeminal neuralgia: the ignition hypothesis. Clin. J. Pain. 18, 4-13 (2002).

85. Barrot, M. Tests and models of nociception and pain in rodents. Neuroscience 211, 39-50 (2012).

86. Blum, E., Procacci, P., Conte, V., Sartori, P. \& Hanani, M Long term effects of lipopolysaccharide on satellite glial cells in mouse dorsal root ganglia. Exp. Cell Res. 350, 236-241 (2017).

87. Peters, C. M. et al. Intravenous paclitaxel administration in the rat induces a peripheral sensory neuropathy characterized by macrophage infiltration and injury to sensory neurons and their supporting cells. Exp. Neurol. 203, 42-54 (2007).

88. Wang, S. et al. P2Y12 shRNA treatment decreases SGC activation to relieve diabetic neuropathic pain in type 2 diabetes mellitus rats. J. Cell Physiol. 233 9620-9628 (2018).

89. Hanani, M., Blum, E., Liu, S., Peng, L. \& Liang, S. Satellite glial cells in dorsal root ganglia are activated in streptozotocin-treated rodents. J. Cell Mol. Med. 18, 2367-2371 (2014)

90. Dantzer, R. O'Connor, J. C. Freund G G Johnson, R. W. \& Kelley, K. W. From inflammation to sickness and depression: when the immune system subjugates the brain. Nat. Rev. Neurosci. 9, 46-56 (2008).

91. Leshchenko, Y. et al. Carbenoxolone does not cross the blood brain barrier: an HPLC study. BMC Neurosci. 7, 3 (2006)

92. Haroutiunian, S., Nikolajsen, L., Finnerup, N. B. \& Jensen, T. S. The neuropathic component in persistent postsurgical pain: a systematic literature review. Pain 154, 95-102 (2013)

93. Steyaert, A. \& De Kock, M. Chronic postsurgical pain. Curr. Opin. Anaesthesiol. 25, 584-588 (2012).

94. Wylde, V et al. Systematic review of management of chronic pain after surgery. Brit. J. Surg. 104, 1293-1306 (2017).

95. Flatters, S. J. Characterization of a model of persistent postoperative pain evoked by skin/muscle incision and retraction (SMIR). Pain 135, 119-130 (2008).

96. Woolf, C. J. Central sensitization: implications for the diagnosis and treatment of pain. Pain 152, S2-S15 (2011).

97. Romero, A Romero-Alejo, E Vasconcelos, N \& Puig, M. M. Glial cell activation in the spinal cord and dorsal root ganglia induced by surgery in mice. Eur. J. Pharmacol. 702, 126-134 (2013).

98. Song, J. et al. The role of P2X7R/ERK signaling in dorsal root ganglia satellite glial cells in the development of chronic postsurgical pain induced by skin/muscle incision and retraction (SMIR). Brain Behav. Immun. 69, 180-189 (2018).

99. Yamakita, S. et al. Synergistic activation of ERK $1 / 2$ between A-fiber neurons and glial cells in the DRC contributes to pain hypersensitivity after tissue injury. Mol. Pain. 14, 1744806918767508 (2018).

100. Pogatzki, E. M., Vandermeulen, E. P. \& Brennan, T. J. Effect of plantar local anesthetic injection on dorsal horn neuron activity and pain behaviors caused by incision. Pain 97, 151-156 (2002).

101. Yatziv, S. L. \& Devor, M. Suppression of neuropathic pain by selective silencing of dorsal root ganglion ectopia using nonblocking concentrations of lidocaine. Pain 160, 2105-2114 (2019).

102. Feldman, E. L., Nave, K A , Jensen, T S \& Bennett, D. L. H. New horizons in diabetic neuropathy: mechanisms, bioenergetics, and pain. Neuron 93 1296-1313 (2017).

103. Tesfaye, S. \& Selvarajah, D. Advances in the epidemiology, pathogenesis and management of diabetic peripheral neuropathy. Diabetes Metab. Res. Rev. 28, 8-14 (2012)

104. Gonçalves, N. P., Vægter, C. B. \& Pallesen, L. T. Peripheral glial cells in the development of diabetic neuropathy. Front. Neurol 9, 268 (2018).

105. Rahman, M. H. et al. Pyruvate dehydrogenase kinasemediated glycolytic metabolic shift in the dorsal root ganglion drives painful diabetic neuropathy. J. Biol. Chem. 291, 6011-6025 (2016).
106. Verkhratsky, A. \& Fernyhough, P. Calcium signalling in sensory neurones and peripheral glia in the contex of diabetic neuropathies. Cell Calcium 56, 362-371 (2014).

107. Hong, S., Morrow, T. J., Paulson, P. E., Isom, L. L. $\&$ Wiley, J. W. Early painful diabetic neuropathy is associated with differential changes in tetrodotoxinsensitive and -resistant sodium channels in dorsal root ganglion neurons in the rat. J. Biol. Chem. 279 29341-29350 (2004)

108. Zochodne, D. W. Diabetic polyneuropathy: an update. Curr. Opin. Neurol. 21, 527-533 (2008).

109. Brownlee, M. \& Cerami, A. The biochemistry of the complications of diabetes mellitus. Annu. Rev. Biochem. 50, 385-432 (1981).

110. Schemmel, K. E., Padiyara, R. S. \& D'Souza, J. J. Aldose reductase inhibitors in the treatment of diabetic peripheral neuropathy: a review. J. Diabetes Complicat. 24, 354-360 (2010)

111. Jiang, Y., Calcutt, N. A., Ramos, K. M. \& Mizisin, A. P. Novel sites of aldose reductase immunolocalization in normal and streptozotocin-diabetic rats. J. Peripher Nerv. Syst. 11, 274-285 (2006).

112. Huang, Q., Liu, Q. \& Ouyang, D. Sorbinil, an aldose reductase inhibitor, in fighting against diabetic complications. Med. Chem. 15, 3-7 (2019).

113. Liu, S et al IncRNA NONRATT021972 siRNA regulates neuropathic pain behaviors in type 2 diabetic rats through the $\mathrm{P} 2 \mathrm{X} 7$ receptor in dorsal root ganglia. Mol. Brain 9, 44 (2016)

114. Coddou, C., Yan, Z., Obsil, T., Huidobro-Toro, J. P. \& Stojilkovic, S. S. Activation and regulation of purinergic P2X receptor channels. Pharmacol. Rev. 63, 641-683 (2011).

115. Teixeira, J. M. et al. Diabetes-induced neuropathic mechanical hyperalgesia depends on $\mathrm{P} 2 \times 4$ receptor activation in dorsal root ganglia. Neuroscience 398 158-170 (2019)

116. Johnson, R. W. \& Rice, A. S. Clinical practice. Postherpetic neuralgia. N. Engl. J. Med. 371 1526-1533 (2014)

117. Steiner, I. \& Benninger, F. Manifestations of herpes virus infections in the nervous system. Neurol. Clin. 36, 725-738 (2018)

118. Fatahzadeh, M. \& Schwartz, R. A. Human herpes simplex virus infections: epidemiology, pathogenesis, symptomatology, diagnosis, and management. J. Am. Acad. Dermatol. 57, 737-763 (2007).

119. Feller, L., Khammissa, R. A. G., Fourie, J., Bouckaert, M. $\&$ Lemmer, J. Postherpetic neuralgia and trigeminal neuralgia. Pain. Res. Treat. 2017, 1681765 (2017).

120. Zerboni, L., Ku, C. C., Jones, C. D. Zehnder, J. L. \& Arvin, A. M. Varicella-zoster virus infection of human dorsal root ganglia in vivo. Proc. Natl Acad. Sci. USA 102, 6490-6495 (2005)

121. Zerboni, L. \& Arvin, A. Neuronal subtype and satellite cell tropism are determinants of varicella-zoster virus virulence in human dorsal root ganglia xenografts in vivo. PLoS Pathog. 11, e1004989 (2015). This paper shows that both neurons and SGCs are infected by varicella zoster herpesvirus in human DRG grafted into mice.

122. Silva, J. R. et al. Neuroimmune-glia interactions in the sensory ganglia account for the development of acute herpetic neuralgia. J. Neurosci. 237, 6408-6422 (2017).

123. Zerboni, L., Sen, N., Oliver, S. L. \& Arvin, A. M Molecular mechanisms of varicella zoster virus pathogenesis. Nat. Rev. Microbiol. 12, 197-210 (2014).

124. Weed, D. J. \& Nicola, A. V. Herpes simplex virus membrane fusion. Adv. Anat. Embryol. Cell Biol. 223 29-47 (2017).

125. Mangus, L. M. et al. SIV-induced immune activation and metabolic alterations in the dorsal root ganglia during acute infection. J. Neuropathol. Exp. Neurol. 78, 78-87 (2019).

126. Li, Y. C., Bai, W. Z Hirano, N., Hayashida, T $\delta$ Hashikawa, T. Coronavirus infection of rat dorsal root ganglia: ultrastructural characterization of viral replication, transfer, and the early response of satellite cells. Virus Res. 163, 628-635 (2012)

127. Hanani, M. Satellite glial cells in sympathetic and parasympathetic ganglia: in search of function. Brain Res. Brain Res. Rev. 64, 304-327 (2010).

128. Verkhratsky, A. \& Nedergaard, M. Astroglial cradle in the life of the synapse. Philos. Trans. R. Soc. Lond. B. Biol. Sci. 369, 20130595 (2014).

129. Feldman-Goriachnik, R., Wu, B. \& Hanani, M. Cholinergic responses of satellite glial cells in the superior cervical ganglia. Neurosci. Lett. 671, 19-24 (2018). 
130. Feldman-Goriachnik, R. \& Hanani, M. The effects of sympathetic nerve damage on satellite glial cells in the mouse superior cervical ganglion. Auton. Neurosci. 221, 102584 (2019)

131. Enes, J. et al. Satellite glial cells modulate cholinergic transmission between sympathetic neurons. PLOS ONE 15, e0218643 (2020).

This paper shows that SGCs in sympathetic ganglia modulate neuron-to-neuron cholinergic neurotransmission, promote synapse formation and contribute to neuronal survival.

132. Shim, H. Rose, J. Halle, S. \& Shekane, P Complex regional pain syndrome: a narrative review for the practising clinician. Br. J. Anaesth. 123, e424-e433 (2109).

133. McLachlan, E. M., Jänig, W., Devor, M. \& Michaelis, M. Peripheral nerve injury triggers noradrenergic sprouting within dorsal root ganglia. Nature 363 543-546 (1993)

134. Li, A. L., Zhang, J. D., Xie, W., Strong, J. A. \& Zhang, J. M. Inflammatory changes in paravertebral sympathetic ganglia in two rat pain models. Neurosci. Bull. 34, 85-97 (2018).

135. Xie, A. X., Lee, J. J. \& McCarthy, K. D. Ganglionic $\mathrm{GFAP}^{+}$glial $\mathrm{Gq}-\mathrm{GPCR}$ signaling enhances heart functions in vivo. J. C. I. Insight 2, e90565 (2017). This study shows that stimulation of SGCs in sympathetic ganglia influences cardiac functions via actions of SGCs on sympathetic neurons.

136. Fukuda, K., Kanazawa, H., Aizawa, Y., Ardell, J. L. \& Shivkumar, K. Cardiac innervation and sudden cardiac death. Circ. Res. 116, 2005-2019 (2015).

137. Hu, P. \& McLachlan, E. M. Macrophage and lymphocyte invasion of dorsal root ganglia after peripheral nerve lesions in the rat. Neuroscience 112, 23-38 (2002).

138. Ji, R. R., Chamessian, A. \& Zhang, Y. O. Pain regulation by non-neuronal cells and inflammation. Science 354, 572-577 (2016).

139. Shinoda, M., Kubo, A., Hayashi, Y. \& Iwata, K Peripheral and central mechanisms of persistent orofacial pain. Front. Neurosci. 13, 1227 (2019)

140. Carlin, D. Halevi, A. E. Ewan, E. E., Moore, A. M. \& Cavalli, V. Nociceptor deletion of Tsc2 enhances axon regeneration by inducing a conditioning injury response in dorsal root ganglia. eNeuro 6 , ENEURO.0168-19.2019 (2019).
141. Jager, S. E. et al. Changes in the transcriptional fingerprint of satellite glial cells following peripheral nerve injury. Glia 68, 1375-1395 (2020).

142. Haberberger, R. V., Barry, C., Dominguez, N. \& Matusica, D. Human dorsal root ganglia. Front. Cell. Neurosci. 13, 271 (2019)

143. Tongtako, W. Canine dorsal root ganglia satellite glial cells represent an exceptional cell population with astrocytic and oligodendrocytic properties. Sci. Rep. 7, 13915 (2017)

144. Takahashi, M. \& Osumi, N. Identification of a novel type II classical cadherin: rat cadherin 19 is expressed in the cranial ganglia and Schwann cell precursors during development. Dev. Dyn. 232, 200-208 (2005).

145. George, D., Ahrens, P. \& Lambert, S. Satellite glial cells represent a population of developmentally arrested Schwann cells. Glia 66, 1496-1506 (2018).

146. Koike, T., Wakabayashi, T., Mori, T., Hirahara, Y. \& Yamada, H. Sox2 promotes survival of satellite glial cells in vitro. Biochem. Biophys. Res. Commun. 464 269-274 (2015)

147. Arora, D. K et al. Evidence of postnatal neurogenesis in dorsal root ganglion: role of nitric oxide and neuronal restrictive silencer transcription factor. J. Molec. Neurosci. 32, 97-107 (2007).

148. Li, H. Y., Say, E. H. \& Zhou, X. F. Isolation and characterization of neural crest progenitors from adult dorsal root ganglia. Stem Cell 25, 2053-2065 (2007).

149. Fex Svenningsen, A., Colman, D. R. \& Pedraza, L. Satellite cells of dorsal root ganglia are multipotential glial precursors. Neuron Glia Biol. 1, 85-93 (2004).

150. Belzer, V., Shraer, N. \& Hanani, M. Phenotypic changes in satellite glial cells in cultured trigeminal ganglia. Neuron Glia Biol. 6, 237-243 (2010).

151. Weider, M. et al. Elevated in vivo levels of a single transcription factor directly convert satellite glia into oligodendrocyte-like cells. PLoS Genet. 11, e 1005008 (2015).

152. van Velzen, M. et al. Neuron-interacting satellite glial cells in human trigeminal ganglia have an APC phenotype. J. Immunol. 183, 2456-2461 (2009). This paper shows that SGCs in human trigeminal ganglia display some properties of immune cells and have a unique leukocyte phenotype.
153. Wu, H. H. et al. Glial precursors clear sensory neuron corpses during development via Jedi-1, an engulfment receptor. Nat. Neurosci. 12, 1534-1541 (2009).

154. Nadeau, J. R., Wilson-Gerwing, T. D. \& Verge, V. M. Induction of a reactive state in perineuronal satellite glial cells akin to that produced by nerve injury is linked to the level of p75NTR expression in adult sensory neurons. Glia 62, 763-777 (2014).

155. Shinder, V. et al. Structural basis of sympatheticsensory coupling in rat and human dorsal root ganglia following peripheral nerve injury. J. Neurocytol. 28 743-761 (1999)

156. Yoshioka, M. et al. Expression of HIV-1 and interleukin- 6 in lumbosacral dorsal root ganglia of patients with AIDS. Neurology 44, 1120-1130 (1994).

157. Koeppen, A. H., Becker, A. B., Qian, J. \& Feustel, P. J. Friedreich ataxia: hypoplasia of spinal cord and dorsal root ganglia. J. Neuropathol. Exp. Neurol. 76, 101-108 (2017).

Acknowledgements

The authors were supported by the Israel Science Foundation (ISF 508/13 and ISF 1297/18 to M.H.), US-Israel Binational Science Foundation (BSF-2011044 to M.H. and D.C.S.) and NIH (R01NS092786, R01NS092466 and R21NS116892 to D.C.S.).

Author contributions

Both authors wrote the article and reviewed and edited the manuscript before submission.

\section{Competing interests}

The authors declare no competing interests.

Peer review information

Nature Reviews Neuroscience thanks the other anonymous reviewers for their contribution to the peer review of this work.

Publisher's note

Springer Nature remains neutral with regard to jurisdictional claims in published maps and institutional affiliations.

(c) Springer Nature Limited 2020 